\title{
Boundedness criteria for commutators of some sublinear operators in weighted Morrey spaces
}

\section{Xiaoli Chen}

Department of Mathematics, Jiangxi Normal University Nanchang, Jiangxi 330022, P. R. China.

Communicated by Y. Z. Chen

\begin{abstract}
In this paper, we obtain bounded criteria on certain weighted Morrey spaces for the commutators generalized by some sublinear integral operators and weighted Lipschitz functions. We also present bounded criteria for commutators generalized by such sublinear integral operators and weighted BMO function on the weighted Morrey spaces. As applications, our results yield the same bounded criteria for those commutators on the classical weighted Morrey spaces.
\end{abstract}

Keywords: Weighted Morrey space, criteria, commutator, weighted Lipschitz function.

2010 MSC: 42B25, 42B30.

(C)2018 All rights reserved.

\section{Introduction and preliminaries}

Suppose that $T$ is a linear or sublinear operator satisfying, for any $f \in L_{\text {loc }}^{1}\left(\mathbb{R}^{n}\right)$ with compact support and $x \notin$ suppf,

$$
|T f(x)| \leqslant C \int_{\mathbb{R}^{n}} \frac{|\Omega(x-y)|}{|x-y|^{n}}|f(y)| d y,
$$

where $C$ is a constant independent of $f$ and $x$. Condition (1.1) was first introduced by Soria and Weiss in [19] for the simple case $\Omega=1$. It is known that many important operators in harmonic analysis satisfy condition (1.1). These operators include the Calderón-Zygmund operator with homogeneous kernel, Carleson's maximal operator with rough kernel, C. Fefferman's singular multipliers, R. Fefferman's singular integrals, Ricci-Stein's oscillatory singular integrals, the Marcinkiewicz integral, and the Bochner-Riesz mean at the critical index, among many others.

On the other hand, in order to study the operators with fractional nature, we consider another kind of linear or sublinear operator $T_{\mu}(0<\mu<n)$, which satisfies that, for any $f \in L_{\text {loc }}^{1}\left(\mathbb{R}^{n}\right)$,

$$
\left|T_{\mu} f(x)\right| \leqslant C \int_{\mathbb{R}^{n}} \frac{|\Omega(x-y)|}{|x-y|^{n-\mu}}|f(y)| d y,
$$

Email address: littleli_chen@163.com (Xiaoli Chen)

doi: $10.22436 /$ jnsa.011.01.04 
where $C$ is a constant independent of $f$ and $x$. Similarly, condition (1.2) is satisfied by many interesting operators in harmonic analysis, such as fractional integrals with homogeneous kernels, fractional maximal functions and fractional oscillatory singular integrals, etc.

The main purpose of the paper is to study the boundedness on the weighted Morrey space of some commutators of those operators $T$ and $T_{\mu}$ mentioned above. Thus, we first define, in the following, the generalized weighted Morrey space $L_{p, q}^{\alpha, \lambda}\left(\Sigma, \omega_{1}, \omega_{2}\right)$, where $0<q \leqslant p<\infty$ and $\omega_{1}, \omega_{2}$ are weights.

Definition 1.1. Let $\Sigma$ be an open set of $\mathbb{R}^{n}, 1 \leqslant p<\infty, 1<q<\infty, \alpha \in(-\infty, \infty)$ and $\lambda \in(0, \infty)$. Suppose $\omega_{1}, \omega_{2}$ are nonnegative weighted functions. For any $f \in \mathrm{L}_{\text {loc }}^{q}(\Sigma)$, let

$$
\|f\|_{L_{p, q}^{\alpha, \lambda}\left(\Sigma, \omega_{1}, \omega_{2}\right)}=\sup _{B \in \mathbb{R}^{n}} \omega_{1}(B)^{-\lambda}\left(\sum_{k=-\infty}^{0} \omega_{1}\left(E_{k}\right)^{\alpha p}\left\|f \chi_{k}\right\|_{L^{q}\left(\Sigma, \omega_{2}\right)}^{p}\right)^{\frac{1}{p}},
$$

where $B=B\left(x_{0}, d\right)$ denotes a ball with center $x_{0}$ and radius $d, E_{k}=B\left(x_{0}, 2^{k} d\right) \backslash B\left(x_{0}, 2^{k-1} d\right), x_{k}=$ $\chi_{E_{k}}$ for $k \in \mathbb{Z}$ is the characteristic function of the set $E_{k}$ and $\left\|f \chi_{k}\right\|_{L q\left(\Sigma, \omega_{2}\right)}=\left(\int_{\Sigma}|f(x)|^{q} \omega_{2}(x) d x\right)^{\frac{1}{q}}$. $\|f\|_{L_{p, q}^{\alpha, \lambda}\left(\Sigma, \omega_{1}, \omega_{2}\right)}$ denotes the $L_{p, q}^{\alpha, \lambda}\left(\Sigma, \omega_{1}, \omega_{2}\right)$ norm of measurable function $f$. If $\|f\|_{L_{p, q}^{\alpha, \lambda}\left(\Sigma, \omega_{1}, \omega_{2}\right)}<\infty$, we say $L_{p, q}^{\alpha, \lambda}\left(\Sigma, \omega_{1}, \omega_{2}\right)$ the weighted general Morrey space.

We make a few comments on the above definition. Firstly, when $\omega_{1}=\omega_{2}=1\left(\omega_{1}(x) d x=\omega_{2}(x) d x\right.$ is the Lebesgue measure), $\alpha=0, p=q$ and $0<\lambda<1$, then $\operatorname{L}_{p, q}^{\alpha, \lambda}\left(\Sigma, \omega_{1}, \omega_{2}\right)$ is the classical Morrey space $\mathrm{L}^{\mathrm{p}, \lambda}$. If $\alpha=0, \mathrm{p}=\mathrm{q}$ and $\lambda=-\frac{1}{\mathrm{p}}+\frac{1}{\mathrm{q}}$, then the right hand side of (1.3) becomes

$$
\sup _{B \in \mathbb{R}^{n}} \omega_{1}(B)^{\frac{1}{p}-\frac{1}{q}}\left(\int_{B}|f(y)|^{p} \omega_{2}(y) d y\right)^{\frac{1}{p}}:=\|f\|_{L^{p, \frac{1}{q}-\frac{1}{p}}\left(\omega_{1}, \omega_{2}\right)^{\prime}}
$$

which is the weighted Morrey space $M_{\mathrm{q}}^{\mathrm{p}}\left(\omega_{1}, \omega_{2}\right)$ introduced by Nakamura and Sawano in [16]. Secondly, assuming that $\omega_{1}=\omega_{2}=\omega$, then $L_{p, q}^{\alpha, \lambda}\left(\Sigma, \omega_{1}, \omega_{2}\right)$ is the weighted Morrey space $M_{q}^{p}(\omega, \omega)$ studied by Komori and Shirai in [9]. Thirdly, when $\omega_{1}=1$ and $\omega_{2}=\omega$ then $L_{p, q}^{\alpha, \lambda}\left(\Sigma, \omega_{1}, \omega_{2}\right)$ corresponds to the weighed Morrey space $M_{\mathbf{q}}^{p}(d x, \omega)$ introduced by Samko in [17].

We denote

$$
\mathrm{L}^{\mathrm{p}, \lambda}\left(\omega_{1}, \omega_{2}\right)=\left\{\mathrm{f}:\|f\|_{\mathrm{L}^{p, \lambda}\left(\omega_{1}, \omega_{2}\right)}<\infty\right\},
$$

as a weighted Morrey space. Following the monotonicity of $\ell^{p}$ spaces, it is easy to show that, for $p_{1} \leqslant p_{2}$,

$$
\mathrm{L}_{p_{1}, q}^{\alpha, \lambda}\left(\Sigma, \omega_{1}, \omega_{2}\right) \subset \mathrm{L}_{p_{2}, q}^{\alpha, \lambda}\left(\Sigma, \omega_{1}, \omega_{2}\right) .
$$

As the reader sees in the above discussion, the Morrey spaces have already received extensive attentions by many authors, since they are important function spaces playing pivot roles in many mathematical fields. It is well-known that Morrey first introduced the classical Morrey space in [15] to study the local behavior of solutions for second order partial elliptic differential equations (so the space and its many extensions are named "Morrey spaces"). After that, this space and its extensions, including its various weighted settings, play significant roles in the study of regularities of solutions to partial differential equations and the theory of function spaces, see [3, 4] etc. Particularly, in [14], Mizuhara introduced a type of Morrey spaces and discussed the boundedness of Calderón-Zygmund operators on these spaces; in [13], Lu et al. established the boundedness on Morrey spaces for several sublinear operators with rough kernels, as well as for their commutators. Here, we only list a few of them among numerous papers in the literature. On the other hand, there are many research papers concerning the boundedness of the commutators generalized by a BMO function $b$ and integral operators satisfying (1.1) or (1.2) on Morrey spaces. We refer the reader to $[2,8,10,18]$ and the references therein. Motivated by these observations, the aim of this paper is to study the boundedness on generalized weighted Morrey space $L_{p, q}^{\alpha, \lambda}\left(\Sigma, \omega_{1}, \omega_{2}\right)$ for the commutators $[b, T]$ and $\left[b, T_{\mu}\right]$. Below, we review the definition of the $\operatorname{Lip}_{\beta, \omega}^{p}$ space and the $\operatorname{BMO}^{p}(\omega)$ space.

Definition 1.2. We will say that a locally integrable function $b$ belongs to the weighted $\operatorname{Lip}_{\beta, \omega}^{p}$ for $1 \leqslant$ 
$p<\infty, 0<\beta<1$ and $\omega \in A_{\infty}$, if

$$
\|b\|_{\operatorname{Lip}_{\beta, \omega}^{p}}=\sup _{B \ni x} \frac{1}{\omega(B)^{\beta / n}}\left(\frac{1}{\omega(B)} \int_{B}\left|b(x)-b_{B}\right|^{p} \omega(x)^{1-p} d x\right)^{\frac{1}{p}} \leqslant C<\infty,
$$

where the supremum is taken over all balls $B \subset \mathbb{R}^{n}$. Put $\operatorname{Lip}_{\beta, \omega}=\operatorname{Lip}_{\beta, \omega}^{1}$. Obviously, for the case $\omega=1$, then the $\operatorname{Lip}_{\beta, \omega}$ space is the classical $\operatorname{Lip}_{\beta}$ space.

Let $\omega \in A_{1}\left(\mathbb{R}^{n}\right)$. Garcia-Cuerva [5] proved that the norms $\|b\|_{\operatorname{Lip}_{\beta, \omega^{\prime}}^{p}}$, coincide with the norm of $\|\mathrm{b}\|_{\operatorname{Lip}_{\beta, \omega}^{1}}$ are equivalent with respect to different values of $p$ provided that $1 \leqslant p \leqslant \infty$.

Definition 1.3. Let $1 \leqslant p<\infty$ and $\omega$ be a weighted function. A locally integrable function $b$ is said to be in the weighted $\mathrm{BMO}$ space $\mathrm{BMO}^{\mathrm{p}}(\omega)$ if

$$
\|b\|_{B M O^{p}(w)}=\sup _{B \ni x}\left(\frac{1}{\omega(B)} \int_{B}\left|b(x)-b_{B}\right|^{p} \omega(x)^{1-p} d x\right)^{\frac{1}{p}} \leqslant C<\infty,
$$

where $\omega(B)=\int_{B} \omega(y) d y$ and the supremum is taken over all balls $B \subset \mathbb{R}^{n}$.

Let $\omega \in A_{1}\left(\mathbb{R}^{n}\right)$. Garcia-Cuerva [5] proved that the norms $\|b\|_{B M O^{p}(\omega)}$ are equivalent with respect to different values of $p$ provided that $1 \leqslant p \leqslant \infty$.

Now we will state our main results.

Theorem 1.4. Let $\mathrm{C}_{0} \geqslant 1$ be an absolute constant and $\mathrm{T}$ be a sublinear operator satisfying (1.1) for all functions $\mathrm{f} \in \mathrm{L}^{1}\left(\mathbb{R}^{\mathrm{n}}\right)$ with compact support and $\mathrm{x} \notin \mathrm{C}_{0}$ suppf. Suppose $\omega \in \mathrm{A}_{1}, \beta \in(0,1), \mathrm{b} \in \operatorname{Lip}_{\beta, \omega^{\prime}}, 1<\mathrm{p}_{1} \leqslant \mathrm{p}_{2}<$ $\infty, 1<\mathrm{q}_{1}, \mathrm{q}_{2}<\infty, \frac{1}{\mathrm{q}_{2}}=\frac{1}{\mathrm{q}_{1}}-\frac{\beta}{\mathrm{n}}$ and $0<\lambda<\infty$. Let $\Omega$ be homogeneous of degree zero and $\Omega \in \mathrm{L}^{\mathrm{r}}\left(\mathrm{S}^{\mathrm{n}-1}\right)$ and $[\mathrm{b}, \mathrm{T}]$ be bounded from $\mathrm{L}^{\mathrm{q}_{1}}\left(\mathbb{R}^{\mathrm{n}}, \boldsymbol{\omega}\right)$ to $\mathrm{L}^{\mathrm{q}_{2}}\left(\mathbb{R}^{\mathrm{n}}, \mathrm{w}^{1-\mathrm{q}_{2}}\right)$. If $\alpha, \mathrm{q}_{1}, \mathrm{q}_{2}$ and $\mathrm{r}$ satisfy one of the following conditions:

(i) $r>\max \left\{\mathbf{q}_{1}^{\prime}, \frac{\mathbf{q}_{2}^{\prime}(n-1)}{n \delta}\right\}$ and $\lambda-\min \left\{\frac{\delta}{\mathbf{q}_{1}}, \frac{1}{\mathbf{q}_{2}}\right\}<\alpha<\lambda+\min \left\{\frac{1}{\mathbf{q}_{1}^{\prime}}, \frac{\delta}{\mathbf{q}_{2}^{\prime}}\right\}+\frac{1}{n r}-\frac{1}{r}$;

(ii) $r>\max \left\{\mathbf{q}_{2}, \frac{(\mathfrak{n}-1) \mathbf{q}_{1}}{\mathfrak{n} \delta}\right\}$ and $\lambda-\min \left\{\frac{\delta}{\mathbf{q}_{1}}, \frac{1}{\mathbf{q}_{2}}\right\}<\alpha<\lambda+\min \left\{\frac{1}{\mathbf{q}_{1}^{\prime}}, \frac{\delta}{\mathbf{q}_{2}^{\prime}}\right\}$,

then $[\mathrm{b}, \mathrm{T}]$ is bounded from $\mathrm{L}_{\mathbf{p}_{1}, \mathbf{q}_{1}}^{\alpha, \lambda}\left(\mathbb{R}^{n}, \omega, \omega\right)$ to $\mathrm{L}_{\mathbf{p}_{2}, \mathbf{q}_{2}}^{\alpha, \lambda}\left(\mathbb{R}^{n}, \omega, \omega^{1-q_{2}}\right)$.

As a corollary, when $\Omega \in \mathrm{L}^{\infty}$ and $\alpha=0, \mathrm{p}_{1}=\mathrm{q}_{1}, \mathrm{p}_{2}=\mathrm{q}_{2}$, we obtain:

Corollary 1.5. Let $\mathrm{T}$ be a sublinear operator and for all functions $f \in \mathrm{L}^{1}\left(\mathbb{R}^{\mathrm{n}}\right)$ with compact support and $x \notin$ Cosuppf,

$$
|T f(x)| \leqslant C \int_{\mathbb{R}^{n}} \frac{|f(y)|}{|x-y|^{n}} d y,
$$

where $\mathrm{C}_{0} \geqslant 1, \mathrm{C}>0$ are absolute constants. Suppose $\omega \in A_{1}, \beta \in(0,1), \mathrm{b} \in \operatorname{Lip}_{\beta, \omega}, 1<\mathrm{q}_{1}, \mathrm{q}_{2}<\infty$ and $\frac{1}{q_{2}}=\frac{1}{q_{1}}-\frac{\beta}{n}$. If $[\mathrm{b}, \mathrm{T}]$ is bounded from $\mathrm{L}^{\mathrm{q}_{1}}\left(\mathbb{R}^{\mathrm{n}}, \omega\right)$ to $\mathrm{L}^{\mathrm{q}_{2}}\left(\mathbb{R}^{\mathrm{n}}, \omega^{1-\mathrm{q}_{2}}\right)$ and $0<\lambda<\min \left\{\frac{\delta}{\mathrm{q}_{1}}, \frac{1}{\mathrm{q}_{2}}\right\}$, then $[\mathrm{b}, \mathrm{T}]$ is bounded from $\mathrm{L}^{\mathrm{q}_{1}, \lambda}(\omega, \omega)$ to $\mathrm{L}^{\mathrm{q}_{2}, \lambda}\left(\omega, \omega^{1-\mathrm{q}_{2}}\right)$.

The corollary is an extension of a result in [7]. In fact, $\mathrm{Hu}$ and $\mathrm{Gu}$ in [7] proved that the commutator $[b, T]$ of a singular integral $T$ satisfying (1.4) is bounded from $L^{q_{1}}(\omega)$ to $L^{q_{2}}\left(\omega^{1-q_{2}}\right)$, where $b \in \operatorname{Lip}_{\beta, \omega}$. Corollary 1.5 further says that $[b, T]$ is bounded from $L^{q_{1}, \lambda}(\omega, \omega)$ to $L^{q_{2}, \lambda}\left(\omega, \omega^{1-q_{2}}\right)$, so it extends the related result in [7].

When $b \in \operatorname{BMO}(\omega)$, a same argument as Theorem 1.4 yields the following.

Theorem 1.6. Let $\mathrm{C}_{0} \geqslant 1$ be an absolute constant and $\mathrm{T}$ be a sublinear operator satisfying (1.1) for all functions $\mathrm{f} \in \mathrm{L}^{1}\left(\mathbb{R}^{\mathrm{n}}\right)$ with compact support and $\mathrm{x} \notin \mathrm{C}_{0}$ suppf. Suppose $\omega \in \mathrm{A}_{1}, \mathrm{~b} \in \mathrm{BMO}(\omega), 1<\mathrm{q}<\infty$ and 
$0<\lambda<\infty$. Let $\Omega$ be homogeneous of degree zero and $\Omega \in \mathrm{L}^{\mathrm{r}}\left(\mathrm{S}^{\mathrm{n}-1}\right)$ and $[\mathrm{b}, \mathrm{T}]$ be bounded from $\mathrm{L}^{\mathrm{q}}\left(\mathbb{R}^{\mathrm{n}}, \omega\right)$ to $\mathrm{L}^{\mathrm{q}}\left(\mathbb{R}^{\mathrm{n}}, \mathrm{w}^{1-\mathrm{q}}\right)$. If $\alpha, \mathrm{q}$ and $\mathrm{r}$ satisfy one of the following conditions:

(i) $r>\max \left\{q^{\prime}, \frac{q^{\prime}(n-1)}{n \delta}\right\}$ and $\lambda-\frac{\delta}{q}<\alpha<\lambda+\frac{\delta}{q}+\frac{1}{n r}-\frac{1}{r}$;

(ii) $r>\max \left\{q, \frac{(n-1) q}{n \delta}\right\}$ and $\lambda-\frac{\delta}{q}<\alpha<\lambda+\frac{\delta}{q}$,

then $[\mathrm{b}, \mathrm{T}]$ is bounded from $\mathrm{L}_{\mathbf{p}, \mathbf{q}}^{\alpha, \lambda}\left(\mathbb{R}^{\mathrm{n}}, \omega, \omega\right)$ to $\mathrm{L}_{\mathbf{p}, \mathbf{q}}^{\alpha, \lambda}\left(\mathbb{R}^{\mathrm{n}}, \omega, \omega^{1-q}\right)$.

Similar to Corollary 1.5 , when $\Omega \in \mathrm{L}^{\infty}$ and $\alpha=0, \mathrm{p}=\mathrm{q}$, we have:

Corollary 1.7. Let $\mathrm{T}$ be a sublinear operator and for all functions $f \in \mathrm{L}^{1}\left(\mathbb{R}^{\mathrm{n}}\right)$ with compact support and $x \notin$ $\mathrm{C}_{0}$ suppf,

$$
|T f(x)| \leqslant C \int_{\mathbb{R}^{n}} \frac{|f(y)|}{|x-y|^{n}} d y,
$$

where $\mathrm{C}_{0} \geqslant 1, \mathrm{C}>0$ are absolute constants. Suppose $\omega \in \mathrm{A}_{1}, \mathrm{~b} \in \mathrm{BMO}(\omega), 1<\mathrm{q}<\infty$. Let $[\mathrm{b}, \mathrm{T}]$ be bounded from $\mathrm{L}^{\mathrm{q}}\left(\mathbb{R}^{\mathrm{n}}, \omega\right)$ to $\mathrm{L}^{\mathrm{q}}\left(\mathbb{R}^{\mathrm{n}}, \omega^{1-\mathrm{q}}\right)$ and $0<\lambda<\frac{\delta}{\mathrm{q}}$. Then $[\mathrm{b}, \mathrm{T}]$ is bounded from $\mathrm{L}^{\mathrm{q}, \lambda}(\omega, \omega)$ to $\mathrm{L}^{\mathrm{q}, \lambda}\left(\omega, \omega^{1-\mathrm{q}}\right)$.

The following theorems focus on the boundedness of the commutators generalized by some integral operators satisfying (1.2) and a weighted $\operatorname{Lip}_{\beta, \omega}$ function or weighted BMO function.

Theorem 1.8. Suppose $\omega \in A_{1}, b \in \operatorname{Lip}_{\beta, \omega}$. Assume $1<p_{1} \leqslant p_{2}<\infty, 1<q_{1}<\infty, 0<\lambda<\infty, 0<\mu<n$ and $\frac{1}{\mathrm{q}_{2}}=\frac{1}{\mathrm{q}_{1}}-\frac{\beta+\mu}{\mathrm{n}}$. Let $\mathrm{T}_{\mu}$ be a sublinear operator satisfying (1.2) for all functions $\mathrm{f} \in \mathrm{L}^{1}\left(\mathbb{R}^{\mathrm{n}}\right)$ with compact support. Assume that $\Omega$ is homogeneous of degree zero and $\Omega \in \mathrm{L}^{\mathrm{r}}\left(\mathrm{S}^{\mathrm{n}-1}\right)\left(\mathrm{r}>\frac{\mathrm{n}}{\mathrm{n}-\mu}\right)$ and the commutator $\left[\mathrm{b}, \mathrm{T}_{\mu}\right]$ is bounded from $\mathrm{L}^{\mathrm{q}_{1}}\left(\mathbb{R}^{\mathrm{n}}, \omega\right)$ to $\mathrm{L}^{\mathrm{q}_{2}}\left(\mathbb{R}^{\mathrm{n}}, \omega^{1-\left(1-\frac{\mu}{n}\right) \mathrm{q}_{2}}\right)$. If $\alpha, \mathrm{q}_{1}, \mathrm{q}_{2}$ and $\mathrm{r}$ satisfy one of the following conditions:

(i) $r>\max \left\{\mathbf{q}_{1}^{\prime}, \frac{\mathbf{q}_{1}^{\prime}(n-1)}{\left(\mathbf{q}_{1}^{\prime} \beta+n\right) \delta}\right\}$ and $\lambda-\min \left\{\frac{\delta}{\mathbf{q}_{1}}-\frac{\mu}{n}, \frac{1}{\mathbf{q}_{2}}\right\}<\alpha<\lambda+\frac{1}{n r}-\frac{1}{r}+\min \left\{\frac{1}{\mathbf{q}_{1}^{\prime}}, \frac{\delta}{\mathbf{q}_{1}^{\prime}}+\frac{\beta \delta}{n}\right\}$;

(ii) $r>\max \left\{q_{2}, \frac{q_{1}(n-1)}{\left(n \delta-q_{1} \mu\right)}\right\}$ and $\lambda+\frac{1}{r}-\frac{1}{n r}-\min \left\{\frac{\delta}{q_{1}}-\frac{\mu}{n}, \frac{1}{q_{2}}\right\}<\alpha<\lambda+\min \left\{\frac{1}{q_{1}^{\prime}}, \frac{\delta}{q_{1}^{\prime}}+\frac{\beta \delta}{n}\right\}$,

then $\left[\mathrm{b}, \mathrm{T}_{\mu}\right]$ is bounded from $\mathrm{L}_{\mathbf{p}_{1}, \mathbf{q}_{1}}^{\alpha, \lambda}\left(\mathbb{R}^{n}, \omega, \omega\right)$ to $\mathrm{L}_{\mathbf{p}_{2}, \mathbf{q}_{2}}^{\alpha, \lambda}\left(\mathbb{R}^{n}, \omega, \omega^{1-\left(1-\frac{\mu}{n}\right) q_{2}}\right)$.

Following the same line to Corollary 1.5, we have:

Corollary 1.9. Suppose $\omega \in A_{1}, b \in \operatorname{Lip}_{\beta, \omega}, 1<q_{1}<\infty, 0<\mu<n$, and $\frac{1}{q_{2}}=\frac{1}{q_{1}}-\frac{\beta+\mu}{n}$. Assume that $T_{\mu}$ is a sublinear operator and for all function $\mathrm{f} \in \mathrm{L}^{1}\left(\mathbb{R}^{\mathrm{n}}\right)$ with compact support,

$$
\left|T_{\mu} f(x)\right| \leqslant C \int_{\mathbb{R}^{n}} \frac{|f(y)|}{|x-y|^{n-\mu}} d y,
$$

where $C>0$ is a absolute constants. Suppose $0<\lambda<\min \left\{\frac{\delta}{q_{1}}-\frac{\mu}{n}, \frac{1}{q_{2}}\right\}$ and the commutator $\left[b, T_{\mu}\right]$ is bounded from $\mathrm{L}^{\mathrm{q}_{1}}\left(\mathbb{R}^{n}, \omega\right)$ to $\mathrm{L}^{\mathrm{q}_{2}}\left(\mathbb{R}^{n}, \omega^{1-\left(1-\frac{\mu}{n}\right) \mathrm{q}_{2}}\right)$. Then $\left[\mathrm{b}, \mathrm{T}_{\mu}\right]$ is bounded from $\mathrm{L}^{\mathrm{q}_{1}, \lambda}(\omega, \omega)$ to $\mathrm{L}^{\mathrm{q}_{2}, \lambda}\left(\omega, \omega^{1-\left(1-\frac{\mu}{n}\right) \mathrm{q}_{2}}\right)$.

Again, $\mathrm{Hu}$ and $\mathrm{Gu}$ in [7] proved that the commutator $\left[b, T_{\mu}\right]$ of a fractional integral $T_{\mu}$ satisfying (1.5), where $b \in \operatorname{Lip}_{\beta, \omega}$ is bounded from $L^{q_{1}}(\omega)$ to $L^{q_{2}}\left(\omega^{\left(1-\frac{\mu}{n}\right)} q_{2}\right)$. Corollary 1.9 further states that $[b, T]$ is bounded from $L^{q_{1}, \lambda}(\omega, \omega)$ to $L^{q_{2}, \lambda}\left(\omega, \omega^{\left(1-\frac{\mu}{n}\right)} q_{2}\right)$.

Similarly to Theorem 1.8, we have:

Theorem 1.10. Suppose $\omega \in A_{1}, b \in \operatorname{BMO}(\omega), 1<p_{1} \leqslant p_{2}<\infty, 1<q_{1}<\infty, 0<\lambda<\infty, 0<\mu<n$ and $\frac{1}{q_{2}}=\frac{1}{q_{1}}-\frac{\mu}{n}$. Let $T_{\mu}$ be a sublinear operator satisfying (1.2) for all functions $f \in L^{1}\left(\mathbb{R}^{n}\right)$ with compact support. Assume that $\Omega$ is homogeneous of degree zero and $\Omega \in \mathrm{L}^{\mathrm{r}}\left(\mathrm{S}^{\mathrm{n}-1}\right)\left(\mathrm{r}>\frac{\mathrm{n}}{\mathrm{n}-\mu}\right)$, the commutator $\left[\mathrm{b}, \mathrm{T}_{\mu}\right]$ is bounded from $\mathrm{L}^{\mathrm{q}_{1}}\left(\mathbb{R}^{\mathrm{n}}, \omega\right)$ to $\mathrm{L}^{\mathrm{q}_{2}}\left(\mathbb{R}^{\mathrm{n}}, \omega^{1-\left(1-\frac{\mu}{n}\right) \mathrm{q}_{2}}\right)$. If $\alpha, \mathrm{q}_{1}, \mathrm{q}_{2}$ and $\mathrm{r}$ satisfy one of the following conditions: 
(i) $r>\max \left\{q_{1}^{\prime}, \frac{\mathbf{q}_{1}^{\prime}(n-1)}{\left(\mathbf{q}_{1}^{\prime}+n\right) \delta}\right\}$ and $\lambda-\min \left\{\frac{\delta}{q_{1}}-\frac{\mu}{n}, \frac{1}{q_{2}}\right\}<\alpha<\lambda+\frac{1}{n r}-\frac{1}{r}+\min \left\{\frac{1}{\mathbf{q}_{1}^{\prime}}, \frac{\delta}{\mathbf{q}_{1}^{\prime}}+\frac{\delta}{n}\right\}$;

(ii) $r>\max \left\{q_{2}, \frac{q_{1}(n-1)}{\left(n \delta-q_{1} \mu\right)}\right\}$ and $\lambda+\frac{1}{r}-\frac{1}{n r}-\min \left\{\frac{\delta}{q_{1}}-\frac{\mu}{n}, \frac{1}{q_{2}}\right\}<\alpha<\lambda+\min \left\{\frac{1}{q_{1}^{\prime}}, \frac{\delta}{q_{1}^{\prime}}+\frac{\delta}{n}\right\}$,

then $\left[\mathrm{b}, \mathrm{T}_{\mu}\right]$ is bounded from $\mathrm{L}_{\mathbf{p}_{1}, \mathbf{q}_{1}}^{\alpha, \lambda}\left(\mathbb{R}^{n}, \omega, \omega\right)$ to $\mathrm{L}_{\mathbf{p}_{2}, \mathbf{q}_{2}}^{\alpha, \lambda}\left(\mathbb{R}^{n}, \omega, \omega^{1-\left(1-\frac{\mu}{n}\right) q_{2}}\right)$.

If $\Omega \in \mathrm{L}^{\infty}$ and $\alpha=0, \mathrm{p}_{1}=\mathrm{q}_{1}, \mathrm{p}_{2}=\mathrm{q}_{2}$, then we have:

Corollary 1.11. Suppose $\omega \in A_{1}, b \in \operatorname{BMO}(\omega), 1<q_{1}<\infty, 0<\mu<n$ and $\frac{1}{q_{2}}=\frac{1}{q_{1}}-\frac{\mu}{n}$. Let $T_{\mu}$ be a sublinear operator satisfying (1.2) for all functions $f \in \mathrm{L}^{1}\left(\mathbb{R}^{\mathrm{n}}\right)$ with compact support. Assume that

$$
0<\lambda<\min \left\{\frac{\delta}{q_{1}}-\frac{\mu}{n}, \frac{1}{q_{2}}\right\}
$$

and the commutator $\left[\mathrm{b}, \mathrm{T}_{\mu}\right]$ is bounded from $\mathrm{L}^{\mathrm{q}_{1}}\left(\mathbb{R}^{\mathrm{n}}, \omega\right)$ to $\mathrm{L}^{\mathrm{q}_{2}}\left(\mathbb{R}^{\mathrm{n}}, \omega^{1-\left(1-\frac{\mu}{n}\right) \mathbf{q}_{2}}\right)$. Then $\left[\mathrm{b}, \mathrm{T}_{\mu}\right]$ is bounded from $\mathrm{L}^{\mathrm{q}_{1}, \lambda}(\omega, \omega)$ to $\mathrm{L}^{\mathrm{q}_{2}, \lambda}\left(\omega, \omega^{1-\left(1-\frac{\mu}{n}\right) \mathbf{q}_{2}}\right)$.

We should remark that $\delta$ in the above theorems or corollaries is the constant in Lemma 2.3 (see the next section). Also, it is known that the commutators $\left[b, T_{\mu}\right]$ are dominated by the fractional integral operator if $b$ is a Lipschitz function so its boundedness can be trivially deduced from known results of the fractional integral. In our case, $b$ is a weighted Lipschitz function and the situation becomes quite different. The reader can check this fact from the definition of weighted Lipschitz function $b$. In other words, to prove Theorem 1.4 and Theorem 1.8, we are not able to use the original approach (for instance, the Stein-Fefferman Sharp maximal function) so we need to seek new methods. For this reason, our computations and estimates in proofs are complicated and possibly very technical.

Remark 1.12. In our knowledge the bounded criteria on the commutators generalized by some sublinear operator and weighted Lipschitz function on Morrey spaces is first introduced. We would also like to remark that our results are new even in the classical weighted Morrey space. On the other hand, we do not need to establish the pointwise estimate for the sharp maximal function of those commutators. For example, the authors [11] obtained the boundedness of the commutator of strongly singular CalderónZygmund operator on Morrey spaces heavily depending on the pointwise estimates for sharp maximal function for such commutators. Comparing with un-weighted case, our computation and proof are more complex and more delicate.

This article is arranged as follows. In Section 2, we will give some preliminaries and some key lemmas. Section 3 presents the proof of Theorems 1.4 and 1.6. Theorems 1.8 and1.10 will be proved in Section 4 .

\section{Preliminary}

In this section, we will provide some information on the $A_{p}$ weight and its related properties used in this article. The following Definition 2.1 is referred to [6].

Definition 2.1. Let $1<p<\infty$. We say $\omega \in A_{p}$ if there exists a constant $C$ such that

$$
\left(\frac{1}{|B|} \int_{B} \omega(x) d x\right)\left(\frac{1}{|B|} \int_{B} \omega(x)^{1-p^{\prime}} d x\right)^{p-1} \leqslant C<\infty
$$

holds for $B \subset \mathbb{R}^{n}$. If there exists a constant $C$ such that

$$
\frac{1}{|B|} \int_{B} \omega(x) d x \leqslant C \omega(x), \text { a.e. } x \in \mathbb{R}^{n},
$$

then we say $\omega \in A_{1}$. 
The following lemma can be found in [12].

Lemma 2.2. Let $\Omega \in \mathrm{L}^{\mathrm{r}}\left(\mathrm{S}^{\mathrm{n}-1}\right)$. Then we have

(i) If $x \in E_{k}, j \leqslant k-2$, then $\int_{E_{j}}|\Omega(x-y)|^{r} d y \leqslant\|\Omega\|_{L^{r}\left(S^{n-1}\right)}^{r} 2^{k(n-1)+j}$;

(ii) if $y \in E_{j}, j \leqslant k-2$, then $\int_{E_{k}}|\Omega(x-y)|^{r} d x \leqslant\|\Omega\|_{L^{r}\left(S^{n-1}\right)}^{r} 2^{k n}$;

(iii) if $x \in E_{k}, j \geqslant k+2$, then $\int_{E_{j}}|\Omega(x-y)|^{r} d y \leqslant\|\Omega\|_{L^{r}\left(S^{n-1}\right)}^{r} 2^{j n}$;

(iv) if $y \in E_{j}, j \geqslant k+2$, then $\int_{E_{k}}|\Omega(x-y)|^{r} d x \leqslant\|\Omega\|_{L^{r}\left(S^{n-1}\right)}^{r} 2^{j(n-1)+k}$.

The following Lemma 2.3 can be found in [1].

Lemma 2.3. Let $\omega \in A_{1}$. Then for all measurable subsets $E$ of ball $B$, there exist $C_{1}, C_{2}>0$ and $0<\delta<1$ such that

$$
C_{1} \frac{|E|}{|B|} \leqslant \frac{\omega(E)}{\omega(B)} \leqslant C_{2}\left(\frac{|E|}{|B|}\right)^{\delta} .
$$

If $\omega(x)$ is a constant, then $\delta=1$. If $\omega(x)$ is not a constant, then $0<\delta<1$.

In the following, we denote $B_{j}=B\left(x_{0}, 2^{j} d\right)$.

Lemma 2.4. Let $E_{j}=B_{j} \backslash B_{j-1}, q_{1}>1$ and $\omega \in A_{q_{1}}$. Then

$$
\int_{E_{j}}|f(y)| d y \leqslant\left\|f \chi_{E_{j}}\right\|_{L^{q_{1}}\left(\mathbb{R}^{n}, w\right)}\left|B_{j}\right| w\left(B_{j}\right)^{-\frac{1}{q_{1}}} .
$$

Proof. Using Hölder's inequality and the fact that $\omega \in A_{q_{1}}$, we obtain that

$$
\int_{E_{j}}|f(y)| d y \leqslant\left(\int_{E_{j}}|f(y)|^{q_{1}} \omega(y) d y\right)^{\frac{1}{q_{1}}}\left(\int_{B_{j}} \omega(y)^{-\frac{q_{1}^{\prime}}{q_{1}}} d y\right)^{\frac{1}{q_{1}^{\prime}}} \leqslant\left\|f \chi_{E_{j}}\right\|_{L^{q_{1}}\left(\mathbb{R}^{n}, \omega\right)}\left|B_{j}\right| \omega\left(B_{j}\right)^{-\frac{1}{q_{1}}} .
$$

Lemma 2.5. Let $E_{j}=B_{j} \backslash B_{j-1}, E_{k}=B_{k} \backslash B_{k-1}, q>1$ and $\omega \in A_{q}$. Then we have

(i) When $j>k+2$, then

$$
\omega\left(E_{j}\right) \lesssim 2^{(j-k) n} \omega\left(E_{k}\right),
$$

and

$$
\omega\left(E_{j}\right) \gtrsim 2^{(j-k) n \delta} \omega\left(E_{k}\right) .
$$

(ii) When $j<k-2$, then

$$
\omega\left(E_{j}\right) \gtrsim 2^{(j-k) n} \omega\left(E_{k}\right),
$$

and

$$
\omega\left(E_{j}\right) \lesssim 2^{(j-k) n \delta} \omega\left(E_{k}\right) .
$$

Proof. First, we prove part (i). When $j>k+2$, it is easy to derive the fact $E_{j} \subset B_{j}$ and $E_{k} \subset B_{k} \subset B_{j}$. Hence Lemma 2.3 yields that

$$
\omega\left(E_{j}\right)=\frac{\omega\left(E_{j}\right)}{\omega\left(B_{j}\right)} \omega\left(B_{j}\right) \geqslant C_{1} \frac{\left|E_{j}\right|}{\left|B_{j}\right|} \omega\left(B_{j}\right) \geqslant C_{1} \frac{\left|E_{j}\right|}{\left|B_{j}\right|} \frac{1}{C_{2}}\left(\frac{\left|B_{j}\right|}{\left|E_{k}\right|}\right)^{\delta} \omega\left(E_{k}\right) \gtrsim 2^{(j-k) n \delta} \omega\left(E_{k}\right),
$$

and

$$
\omega\left(E_{j}\right)=\frac{\omega\left(E_{j}\right)}{\omega\left(B_{j}\right)} \omega\left(B_{j}\right) \leqslant C_{2}\left(\frac{\left|E_{j}\right|}{\left|B_{j}\right|}\right)^{\delta} \omega\left(B_{j}\right) \leqslant C_{2}\left(\frac{\left|E_{j}\right|}{\left|B_{j}\right|}\right)^{\delta} \frac{1}{C_{1}} \frac{\left|B_{j}\right|}{\left|E_{k}\right|} \omega\left(E_{k}\right) \lesssim 2^{(j-k) n} \omega\left(E_{k}\right) .
$$


For (ii) of the lemma, when $j<k-2$, we have $E_{j} \subset B_{j}, E_{k} \subset B_{k}$ and $B_{j} \subset B_{k}$. Using Lemma 2.3 again, we have

$$
\begin{aligned}
\omega\left(E_{j}\right) & =\frac{\omega\left(E_{j}\right)}{\omega\left(B_{j}\right)} \omega\left(B_{j}\right) \geqslant C_{1} \frac{\left|E_{j}\right|}{\left|B_{j}\right|} \omega\left(B_{j}\right) \geqslant C_{1} \frac{\left|E_{j}\right|}{\left|B_{j}\right|} C_{1} \frac{\left|B_{j}\right|}{\left|B_{k}\right|} \omega\left(B_{k}\right) \\
& \geqslant C_{1}^{2} \frac{\left|E_{j}\right|}{\left|B_{k}\right|} \frac{1}{C_{2}}\left(\frac{\left|B_{k}\right|}{E_{k} \mid}\right)^{\delta} \omega\left(E_{k}\right) \gtrsim 2^{(j-k) n} \omega\left(E_{k}\right),
\end{aligned}
$$

and

$$
\begin{aligned}
\omega\left(E_{j}\right) & =\frac{\omega\left(E_{j}\right)}{\omega\left(B_{j}\right)} \omega\left(B_{j}\right) \leqslant C_{2}\left(\frac{\left|E_{j}\right|}{\left|B_{j}\right|}\right)^{\delta} \omega\left(B_{j}\right) \leqslant C_{2}\left(\frac{\left|E_{j}\right|}{\left|B_{j}\right|}\right)^{\delta} C_{2}\left(\frac{\left|B_{j}\right|}{\left|B_{k}\right|}\right)^{\delta} \omega\left(B_{k}\right) \\
& \leqslant C_{2}^{2}\left(\frac{\left|E_{j}\right|}{\left|B_{j}\right|}\right)^{\delta}\left(\frac{\left|B_{j}\right|}{\left|B_{k}\right|}\right)^{\delta} \frac{\left|B_{k}\right|}{\left|E_{k}\right|} \omega\left(E_{k}\right) \lesssim 2^{(j-k) n \delta} \omega\left(E_{k}\right),
\end{aligned}
$$

which completes the proof of the lemma.

Lemma 2.6. Let $\mathrm{E}_{j}=\mathrm{B}_{j} \backslash \mathrm{B}_{j-1}$ and $\omega \in \mathrm{A}_{1}$. Then we have

$$
\left(\omega\left(E_{j}\right)^{-p \lambda+\alpha p}\left\|f \chi_{E_{j}}\right\|_{L^{q}\left(\mathbb{R}^{n}, \omega\right)}^{p}\right)^{\frac{1}{p}} \leqslant C\|f\|_{L_{p, q}^{\alpha, \lambda}\left(\mathbb{R}^{n}, \omega, \omega\right)} .
$$

Proof. Let $\bar{B}=B_{j}, \bar{E}_{k}=B_{j+k} \backslash B_{j-1+k}($ for $k \leqslant 0)$. We then have $E_{j}=\bar{E}_{0} \subset \bar{B}$. Thanks to Lemma 2.3, it gives that

$$
\omega\left(E_{j}\right)=\omega\left(\bar{E}_{0}\right) \geqslant C_{1} \omega(\bar{B}) \frac{\left|\overline{\mathrm{E}}_{0}\right|}{|\overline{\mathrm{B}}|} \geqslant \mathrm{C} \omega(\overline{\mathrm{B}}) .
$$

The above estimates together with Definition 1.1 yield that

$$
\begin{aligned}
\left(\omega\left(\mathrm{E}_{j}\right)^{-p \lambda+\alpha p}\left\|f \chi_{E_{j}}\right\|_{L^{q}\left(\mathbb{R}^{n}, \omega\right)}^{p}\right)^{\frac{1}{p}} & =\left(\omega\left(\bar{E}_{0}\right)^{-p \lambda} \omega\left(\bar{E}_{0}\right)^{\alpha p}\left\|f \chi_{\bar{E}_{0}}\right\|_{L^{q}\left(\mathbb{R}^{n}, \omega\right)}^{p}\right)^{\frac{1}{p}} \\
& \leqslant C\left(\omega(\bar{B})^{-p} \lambda \sum_{k=-\infty}^{0} \omega\left(\bar{E}_{k}\right)^{\alpha p}\left\|f \chi_{\bar{E}_{k}}\right\|_{L^{q}\left(\mathbb{R}^{n}, \omega\right)}^{p}\right)^{\frac{1}{p}} \leqslant C\|f\|_{L_{p}^{\alpha, q}\left(\mathbb{R}^{n}, \omega, \omega\right)} .
\end{aligned}
$$

\section{Weighted bounded criteria for the commutator of singular integral type operator}

In this section, we will present the proof of Theorem 1.4. The proof of Theorem 1.6 is similar, so we skip it.

Note that if $p_{1}<p_{2}$, then

$$
\mathrm{L}_{\mathbf{p}_{1}, \mathbf{q}_{2}}^{\alpha, \lambda}\left(\omega, \omega^{1-\left(1-\frac{\mu}{n}\right) q_{2}}\right) \subset L_{\mathbf{p}_{2}, \mathbf{q}_{2}}^{\alpha, \lambda}\left(\omega, \omega^{1-\left(1-\frac{\mu}{n}\right) q_{2}}\right) .
$$

So we need only to prove Theorem 1.4 for the case $p_{1}=p_{2}=p$.

Fix a ball $B=B\left(x_{0}, d\right)$. Let $E_{j}=B\left(x_{0}, 2^{j} d\right) \backslash B\left(x_{0}, 2^{j-1} d\right)$. We write $x_{j}=\chi_{E_{j}}$ for simplicity in the notation. Thus, we may write $f$ as the form

$$
f(y)=\sum_{j=-\infty}^{\infty} f(y) x_{j}(y)=\sum_{j=-\infty}^{\infty} f_{j}(y) .
$$

Furthermore, we have that

$$
\omega(B)^{-p \lambda} \sum_{k=-\infty}^{0} \omega\left(E_{k}\right)^{\alpha p}\left\|[b, T] f \chi_{k}\right\|_{L^{q_{2}}\left(\mathbb{R}^{n}, \omega^{1-q_{2}}\right)}^{p}
$$




$$
\begin{aligned}
& \leqslant \omega(B)^{-p \lambda} \sum_{k=-\infty}^{0} \omega\left(E_{k}\right)^{\alpha p}\left(\sum_{j=-\infty}^{k-2}\left\|[b, T] f_{j} \chi_{k}\right\|_{L^{q_{2}}\left(\mathbb{R}^{n}, \omega^{1-q_{2}}\right)}\right)^{p} \\
& +\omega(B)^{-p \lambda} \sum_{k=-\infty}^{0} \omega\left(E_{k}\right)^{\alpha p}\left(\sum_{j=k-1}^{k+1}\left\|[b, T] f_{j} \chi_{k}\right\|_{L^{q_{2}\left(\mathbb{R}^{n}, \omega^{\left.1-q_{2}\right)}\right.}}\right)^{p}
\end{aligned}
$$

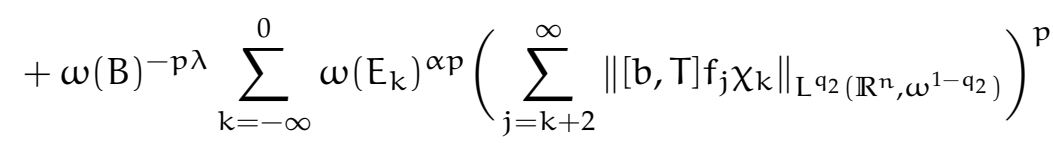

$$
\begin{aligned}
& =: I_{1}+I_{2}+I_{2} \text {. }
\end{aligned}
$$

Firstly, the $\left(\mathrm{L}^{\mathrm{q}_{1}}\left(\mathbb{R}^{\mathrm{n}}, \omega\right), \mathrm{L}^{\mathrm{q}_{2}}\left(\mathbb{R}^{\mathrm{n}}, \omega^{1-\mathrm{q}_{2}}\right)\right)$-boundedness of commutator $[\mathrm{b}, \mathrm{T}]$ yields that

$$
I_{2} \leqslant \omega(B)^{-p \lambda} \sum_{k=-\infty}^{0} \omega\left(E_{k}\right)^{\alpha p}\left(\sum_{j=k-1}^{k+1}\left\|f_{j} \chi_{k}\right\|_{L^{q_{1}}\left(\mathbb{R}^{n}, \omega\right)}\right)^{p} \leqslant C\|f\|_{L_{p, q_{1}}^{\alpha, \lambda}\left(\mathbb{R}^{n}, \omega, \omega\right)}^{p} .
$$

To estimate $I_{1}$, when $x \in E_{k}, y \in E_{j}, j \leqslant k-2$, one can easily deduce $|x-y| \sim\left|x-x_{0}\right| \sim 2^{k} d$. If $q_{2}<r$, we obtain, by Minkowski's inequality that

$$
\begin{aligned}
\left\|[\mathrm{b}, \mathrm{T}] \mathrm{f}_{j} \chi_{k}\right\|_{\mathrm{L}_{2}\left(\mathbb{R}^{n}, w^{\left.1-\mathrm{q}_{2}\right)} \leqslant\right.} & C 2^{-k n}\left(\int_{E_{k}}\left(\int_{E_{j}}|\Omega(x-y)\|b(x)-b(y)\| f(y)| d y\right)^{q_{2}} \omega^{1-q_{2}}(x) d x\right)^{\frac{1}{q_{2}}} \\
\leqslant & C 2^{-k n} \int_{E_{j}}\left(\int_{E_{k}}|\Omega(x-y)|^{q_{2}}\left|b(x)-b_{B_{k}}\right|^{q_{2}} w^{1-q_{2}}(x) d x\right)^{\frac{1}{q_{2}}}|f(y)| d y \\
& +C 2^{-k n} \int_{E_{j}}\left(\int_{E_{k}}|\Omega(x-y)|^{q_{2}} \omega^{1-q_{2}}(x) d x\right)^{\frac{1}{q_{2}}}\left|b_{B_{k}}-b(y) \| f(y)\right| d y \\
:= & C\left(U_{1}+U_{2}\right) .
\end{aligned}
$$

Choose $s>\max \left\{q_{2}, q_{1}^{\prime}\right\}$. Then applying Hölder's inequality, (ii) of Lemma 2.2, the reverse Hölder inequality, the property of $\omega \in A_{q_{1}^{\prime}}$ and Lemma 2.4 , we can obtain

$$
\begin{aligned}
& \mathrm{U}_{1} \leqslant \mathrm{C} 2^{-\mathrm{kn}} \int_{\mathrm{E}_{\mathrm{j}}}\left(\int_{\mathrm{E}_{\mathrm{k}}}|\Omega(x-y)|^{\mathrm{r}} \mathrm{d} x\right)^{\frac{1}{\mathrm{r}}}\left(\int_{\mathrm{B}_{\mathrm{k}}}\left|\mathrm{b}(x)-\mathrm{b}_{\mathrm{B}_{\mathrm{k}}}\right|^{s} \omega^{1-s}(x) \mathrm{d} x\right)^{\frac{1}{s}}
\end{aligned}
$$

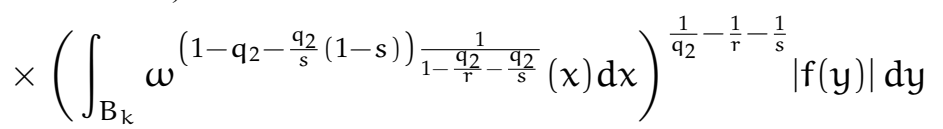

$$
\begin{aligned}
& \leqslant C 2^{-k n}\|\Omega\|_{L^{r}\left(S^{n-1}\right)} 2^{\frac{k n}{r}}\|b\|_{L i p_{\beta, \omega}^{s}} \omega\left(B_{k}\right)^{\frac{\beta}{n}+\frac{1}{s}} \omega\left(B_{k}\right)^{\frac{1}{q_{2}}-\frac{1}{s}}\left|B_{k}\right|^{-\frac{1}{r}} \int_{E_{j}}|f(y)| d y \\
& \leqslant C 2^{-k n}\|\Omega\|_{L^{r}\left(S^{n-1}\right)}\|b\|_{\operatorname{Lip}_{\beta, \omega}^{s}} \omega\left(B_{k}\right)^{\frac{\beta}{n}+\frac{1}{q_{2}}}\left\|f \chi_{E_{j}}\right\|_{L^{q_{1}}\left(\mathbb{R}^{n}, \omega\right)}\left|B_{j}\right| \omega\left(B_{j}\right)^{-\frac{1}{q_{1}}}
\end{aligned}
$$

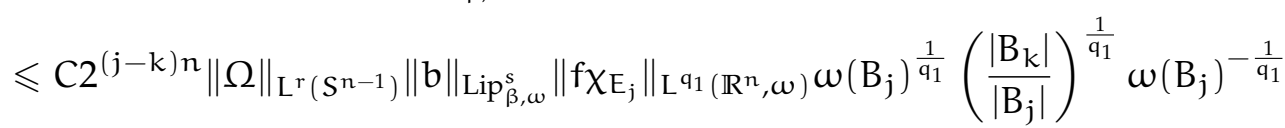

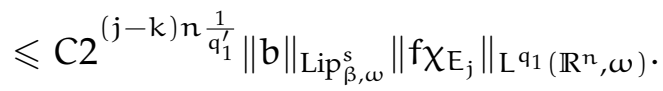

Thanks to Hölder's inequality and the fact that $\omega \in A_{\frac{\mathrm{q}_{2}^{\prime}}{\mathrm{r}^{\prime}}}$, we have that

$$
\mathrm{U}_{2} \leqslant \mathrm{C} 2^{-\mathrm{kn}} \int_{\mathrm{E}_{j}}\left(\int_{\mathrm{E}_{k}}|\Omega(x-y)|^{\mathrm{r}} \mathrm{d} x\right)^{\frac{1}{\mathrm{r}}}\left(\int_{\mathrm{B}_{k}} \omega^{\left(1-\mathrm{q}_{2}\right)\left(\frac{\mathrm{r}}{\mathrm{q}_{2}}\right)^{\prime}}(x) \mathrm{d} x\right)^{\frac{1}{\mathrm{q}_{2}}-\frac{1}{\mathrm{r}}}\left|\mathrm{b}_{\mathrm{B}_{k}}-b(y) \| f(y)\right| d y
$$




$$
\begin{aligned}
& \leqslant C 2^{-k n}\|\Omega\|_{L^{r}\left(S^{n-1}\right)} 2^{\frac{k n}{r}} \omega\left(B_{k}\right)^{\frac{1}{q_{2}}-1}\left|B_{k}\right|^{1-\frac{1}{r}}\left(\int_{E_{j}}|f(y)|^{q_{1}} \omega d y\right)^{\frac{1}{q_{1}}} \\
& \times\left(\int_{B_{j}}\left|b_{B_{k}}-b(y)\right|^{s} \omega(y)^{1-s} d y\right)^{\frac{1}{s}}\left(\int_{B_{j}} \omega(y)^{\left(\frac{s-1}{s}-\frac{1}{q_{1}}\right) \frac{1}{1-\frac{1}{q_{1}}-\frac{1}{s}}} d y\right)^{1-\frac{1}{q_{1}}-\frac{1}{s}} \\
& \leqslant C\|\Omega\|_{L^{r}\left(S^{n-1}\right)} \omega\left(B_{k}\right)^{\frac{1}{q_{2}}-1}\left\|f \chi_{E_{j}}\right\|_{L^{q_{1}}\left(\mathbb{R}^{n}, \omega\right)} \omega\left(B_{j}\right)^{\frac{\beta}{n}+\frac{1}{s}}(k-j)\|b\|_{L_{\beta, \omega}^{s}} \omega\left(B_{j}\right)^{1-\frac{1}{q_{1}}-\frac{1}{s}}
\end{aligned}
$$

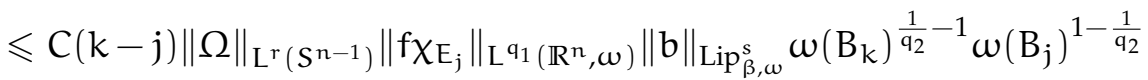

$$
\begin{aligned}
& \leqslant C(k-j)\|\Omega\|_{L^{r}\left(S^{n-1}\right)}\left\|f \chi_{E_{j}}\right\|_{L^{q_{1}}\left(\mathbb{R}^{n}, \omega\right)}\|b\|_{L_{\beta, \omega}^{s}} \omega\left(B_{k}\right)^{\frac{1}{q_{2}}-1} \omega\left(B_{k}\right)^{1-\frac{1}{q_{2}}} 2^{(j-k) n \delta\left(1-\frac{1}{q_{2}}\right)} \\
& \leqslant C(k-j) 2^{(j-k) \frac{n \delta}{q_{2}^{\prime}}}\left\|f \chi_{E_{j}}\right\|_{L^{q_{1}}\left(\mathbb{R}^{n}, \omega\right)}
\end{aligned}
$$

which, along with the estimate of $U_{1}$, gives that

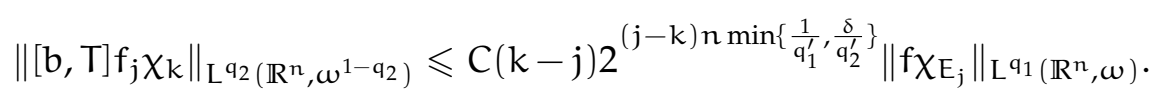

Now, from Lemma 2.6 and (ii) of Lemma 2.5, it follows that

$$
\begin{aligned}
& I_{1} \leqslant C \omega(B)^{-p \lambda} \sum_{k=-\infty}^{0} \omega\left(E_{k}\right)^{\alpha p}\left(\sum_{j=-\infty}^{k-2}(k-j) 2^{(j-k) n \min \left\{\frac{1}{q_{1}^{\prime}}, \frac{\delta}{q_{2}^{\prime}}\right\}}\left\|f \chi_{E_{j}}\right\|_{L^{q_{1}}\left(\mathbb{R}^{n}, \omega\right)}\right)^{p} \\
& \leqslant \mathrm{C} \omega(\mathrm{B})^{-p \lambda} \sum_{k=-\infty}^{0} \omega\left(E_{k}\right)^{\alpha p}\left(\sum_{j=-\infty}^{k-2}(k-j) 2^{(j-k) n \min \left\{\frac{1}{q_{1}^{\prime}}, \frac{\delta}{q_{2}^{\prime}}\right\}} \omega\left(E_{j}\right)^{-\alpha+\lambda}\right. \\
& \left.\times\left(\omega\left(E_{j}\right)^{-\lambda p+\alpha p}\left\|f \chi_{E_{j}}\right\|_{L^{q_{1}}\left(\mathbb{R}^{n}, \omega\right)}^{p}\right)^{\frac{1}{p}}\right)^{p} \\
& \leqslant C\|f\|_{L_{p, q_{1}}^{\alpha, \lambda}\left(\mathbb{R}^{n}, \omega, \omega\right)}^{p} \omega(B)^{-p \lambda} \sum_{k=-\infty}^{0} \omega\left(E_{k}\right)^{\alpha p}
\end{aligned}
$$

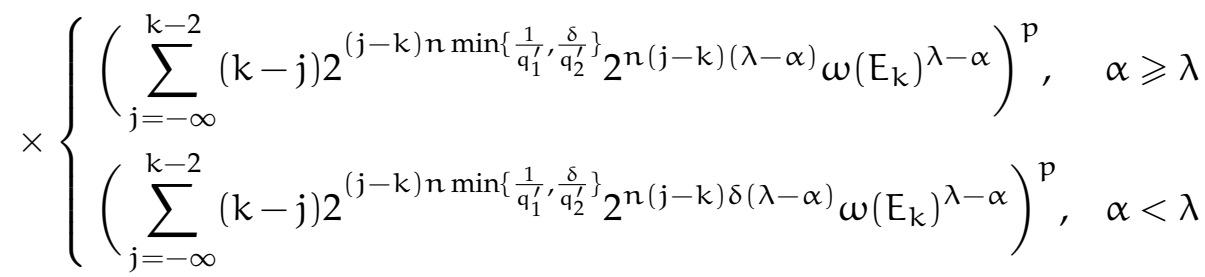

$$
\begin{aligned}
& \leqslant C\|f\|_{L_{p, q_{1}}^{\alpha, \lambda}\left(\mathbb{R}^{n}, \omega, \omega\right)}^{p} \omega(B)^{-p \lambda} \sum_{k=-\infty}^{0} \omega\left(E_{k}\right)^{\lambda p} \\
& \times\left\{\begin{array}{l}
\left(\sum_{j=-\infty}^{k-2}(k-j) 2^{(j-k) n\left(\min \left\{\frac{1}{q_{1}^{\prime}}, \frac{\delta}{q_{2}^{\prime}}\right\}+\lambda-\alpha\right)}\right)^{p}, \quad \alpha \geqslant \lambda \\
\left(\sum_{j=-\infty}^{k-2}(k-j) 2^{(j-k) n\left(\min \left\{\frac{1}{q_{1}^{\prime}}, \frac{\delta}{q_{2}^{\prime}}\right\}+\lambda \delta-\alpha \delta\right)}\right)^{p}, \quad \alpha<\lambda
\end{array}\right. \\
& \leqslant C\|f\|_{L_{p, q_{1}}^{\alpha, \lambda}\left(\mathbb{R}^{n}, w, w\right)^{\prime}}^{p}
\end{aligned}
$$

where we require either $\lambda \leqslant \alpha<\lambda+\min \left\{\frac{1}{\mathbf{q}_{1}^{\prime}}, \frac{\delta}{\mathbf{q}_{2}^{\prime}}\right\}$ or $\alpha<\lambda$.

If $r>\max \left\{q_{1}^{\prime}, \frac{(n-1) q_{2}^{\prime}}{n \delta}\right\}$, then we write

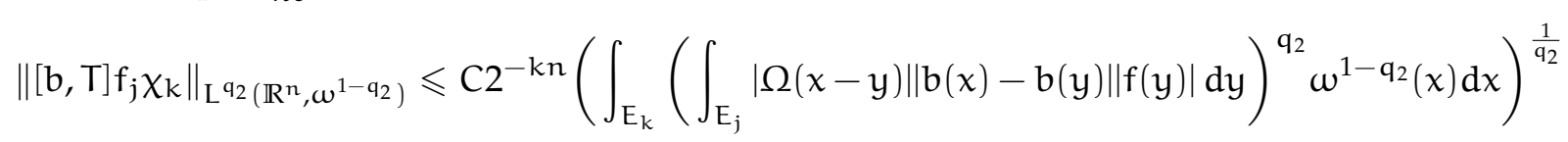




$$
\begin{aligned}
\leqslant & C 2^{-k n}\left(\int_{E_{k}}\left|b(x)-b_{B_{k}}\right|^{q_{2}}\left(\int_{E_{j}}|\Omega(x-y) \| f(y)| d y\right)^{q_{2}} w^{1-q_{2}}(x) d x\right)^{\frac{1}{q_{2}}} \\
& +C 2^{-k n}\left(\int_{E_{k}}\left(\int_{E_{j}}\left|\Omega(x-y)\left\|b_{B_{k}}-b(y)\right\| f(y)\right| d y\right)^{q_{2}} \omega^{1-q_{2}}(x) d x\right)^{\frac{1}{q_{2}}} \\
:= & C\left(U_{1}^{\prime}+U_{2}^{\prime}\right) .
\end{aligned}
$$

To estimate term $U_{1}^{\prime}$, we use Hölder's inequality, (i) of Lemma 2.2 and the property of $\omega \in A_{\frac{q_{1}}{r^{\prime}}}$ to obtain that

$$
\begin{aligned}
& \mathrm{u}_{1}^{\prime} \leqslant \mathrm{C} 2^{-\mathrm{kn}}\left(\int _ { \mathrm { E } _ { \mathrm { k } } } | \mathrm { b } ( \mathrm { x } ) - \mathrm { b } _ { \mathrm { B } _ { \mathrm { k } } } | ^ { \mathrm { q } _ { 2 } } \left(\left(\int_{\mathrm{E}_{\mathrm{j}}}|\Omega(x-y)|^{\mathrm{q}_{1}^{\prime}} \boldsymbol{w}(\mathrm{y})^{-\frac{\mathrm{q}_{1}^{\prime}}{\mathrm{q}_{1}}} \mathrm{dy}\right)^{\frac{1}{\mathrm{q}_{1}^{\prime}}}\right.\right. \\
& \left.\left.\times\left(\int_{E_{j}}|f(y)|^{q_{1}} w(y) d y\right)^{\frac{1}{q_{1}}}\right)^{q_{2}} \omega^{1-q_{2}}(x) d x\right)^{\frac{1}{q_{2}}} \\
& \leqslant C 2^{-k n}\left\|f \chi_{E_{j}}\right\|_{L^{q_{1}}\left(\mathbb{R}^{n}, w\right)}\left(\int _ { E _ { k } } | b ( x ) - b _ { B _ { k } } | ^ { \mathrm { q } _ { 2 } } \left(\left(\int_{E_{j}}|\Omega(x-y)|^{r} d y\right)^{\frac{1}{r}}\right.\right. \\
& \left.\left.\times\left(\int_{B_{j}} \omega(y)^{-\frac{q_{1}^{\prime}}{q_{1}}\left(\frac{r}{q_{1}^{\prime}}\right)^{\prime}} d y\right)^{\frac{1}{q_{1}^{\prime}}-\frac{1}{r}}\right)^{q_{2}} \omega^{1-q_{2}}(x) d x\right)^{\frac{1}{q_{2}}} \\
& \leqslant C 2^{-k n}\left\|f \chi_{E_{j}}\right\|_{L^{q_{1}}\left(\mathbb{R}^{n}, \omega\right)}\|\Omega\|_{L^{r}\left(S^{n-1}\right)} 2^{\frac{k(n-1)}{r}+\frac{j}{r}}\left(\int_{B_{j}} \omega(y)^{-\frac{1}{q_{1}-\frac{q_{1}}{r}-1}} d y\right)^{\frac{1}{q_{1}^{\prime}}-\frac{1}{r}} \\
& \times\left(\int_{B_{k}}\left|b(x)-b_{B_{k}}\right|^{q_{2}} w^{1-q_{2}}(x) d x\right)^{\frac{1}{q_{2}}} \\
& \leqslant C 2^{-k n}\left\|f \chi_{E_{j}}\right\|_{L^{q_{1}}\left(\mathbb{R}^{n}, \omega\right)}\|\Omega\|_{L^{r}\left(S^{n-1}\right)} 2^{\frac{k(n-1)}{r}+\frac{j}{r}} \omega\left(B_{j}\right)^{-\frac{1}{q_{1}}}\left|B_{j}\right|^{1-\frac{1}{r}}\|b\|_{L_{\beta_{\beta}, \omega}^{q_{2}}} \omega\left(B_{k}\right)^{\frac{\beta}{n}+\frac{1}{q_{2}}} \\
& \leqslant C 2^{(j-k)\left(n-\frac{n}{r}+\frac{1}{r}\right)}\left\|f \chi_{E_{j}}\right\|_{L^{q_{1}\left(\mathbb{R}^{n}, \omega\right)}}\|\Omega\|_{L^{r}\left(S^{n-1}\right)} \omega\left(B_{j}\right)^{-\frac{1}{q_{1}}}\|b\|_{\operatorname{Lip}_{\beta, \omega}^{q_{2}}} \omega\left(B_{j}\right)^{\frac{1}{q_{1}}}\left(\frac{\left|B_{k}\right|}{\left|B_{j}\right|}\right)^{\frac{1}{q_{1}}} \\
& \leqslant C 2^{(j-k) n\left(\frac{1}{q_{1}^{\prime}}-\frac{1}{r}+\frac{1}{n r}\right)}\|\Omega\|_{L^{r}\left(S^{n-1}\right)}\left\|f \chi_{E_{j}}\right\|_{L^{q_{1}}\left(\mathbb{R}^{n}, \omega\right)}\|b\|_{\operatorname{Lip}_{\beta, \omega}^{q_{2}}} \text {. }
\end{aligned}
$$

For $\mathrm{U}_{2}^{\prime}$, we choose $s>\frac{\mathrm{q}_{1}^{\prime} \mathrm{r}}{\mathrm{r}-\mathrm{q}_{1}^{\prime}}$. Using Hölder's inequality, the reverse Hölder inequality and (i) of Lemma 2.2 , we have that

$$
\begin{aligned}
& \mathrm{U}_{2}^{\prime}=\mathrm{C} 2^{-\mathrm{kn}}\left(\int_{\mathrm{E}_{\mathrm{k}}}\left(\int_{\mathrm{E}_{j}}\left|\Omega(x-y)\left\|\mathrm{b}_{\mathrm{B}_{\mathrm{k}}}-\mathrm{b}(\mathrm{y})\right\| \mathrm{f}(\mathrm{y})\right| \mathrm{dy}\right)^{\mathrm{q}_{2}} \omega^{1-\mathrm{q}_{2}}(\mathrm{x}) \mathrm{dx}\right)^{\frac{1}{\mathrm{q}_{2}}} \\
& \leqslant C 2^{-k n}\left(\int_{E_{k}}\left(\int_{E_{j}}|\Omega(x-y)|^{q_{1}^{\prime}}\left|b_{B_{k}}-b(y)\right|^{q_{1}^{\prime}} \omega(y)^{-\frac{q_{1}^{\prime}}{q_{1}}} d y\right)^{\frac{q_{2}}{q_{1}^{\prime}}}\right. \\
& \left.\times\left(\int_{E_{j}}|f(y)|^{q_{1}} \omega(y) d y\right)^{\frac{q_{2}}{q_{1}}} \omega(x)^{1-q_{2}} d x\right)^{\frac{1}{q_{2}}} \\
& \leqslant C 2^{-k n}\left\|f \chi_{E_{j}}\right\|_{L^{q_{1}}\left(\mathbb{R}^{n}, w\right)}\left(\int_{E_{k}}\left(\int_{E_{j}}|\Omega(x-y)|^{r} d y\right)^{\frac{q_{2}}{r}}\left(\int_{B_{j}}\left|b(y)-b_{B_{k}}\right|^{s} \omega(y)^{1-s} d y\right)^{\frac{q_{2}}{s}}\right.
\end{aligned}
$$

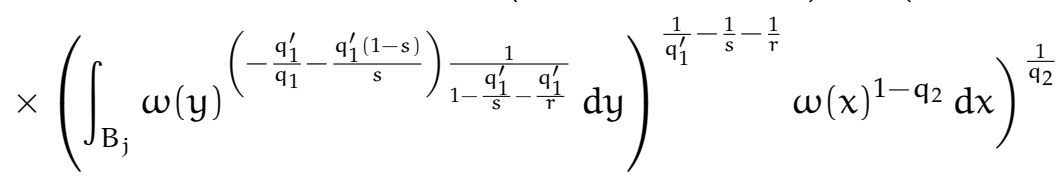




$$
\begin{aligned}
& \leqslant C 2^{-k n} 2^{\frac{k(n-1)+j}{r}}\left\|f \chi_{E_{j}}\right\|_{L^{q_{1}}\left(\mathbb{R}^{n}, w\right)}\|\Omega\|_{L^{r}\left(S^{n-1}\right)}(k-j)\|b\|_{L_{\beta, ~}^{s}, \omega}^{s} \omega\left(B_{j}\right)^{\frac{\beta}{n}+\frac{1}{s}}\left|B_{j}\right|^{-\frac{1}{r}} \omega\left(B_{j}\right)^{\frac{1}{q_{1}^{\prime}}-\frac{1}{s}} \\
& \times\left(\int_{B_{k}} \omega(x)^{1-q_{2}} d x\right)^{\frac{1}{q_{2}}} \\
& \leqslant C(k-j) 2^{(j-k)\left(\frac{1}{r}-\frac{n}{r}\right)} 2^{-k n}\left\|f \chi_{E_{j}}\right\|_{L^{q_{1}}\left(\mathbb{R}^{n}, \omega\right)}\|\Omega\|_{L^{r}\left(S^{n-1}\right)}\|b\|_{L_{p_{3, \omega}^{s}}^{s}} \omega\left(B_{j}\right)^{1-\frac{1}{q_{2}}}\left|B_{k}\right| \omega\left(B_{k}\right)^{\frac{1}{q_{2}}-1} \\
& \leqslant C(k-j) 2^{(j-k) n\left(\frac{1}{n r}-\frac{1}{r}\right)}\|\Omega\|_{L^{r}\left(S^{n-1}\right)}\left\|f \chi_{E_{j}}\right\|_{L^{q_{1}}\left(\mathbb{R}^{n}, w\right)}\|b\|_{\operatorname{Lip}_{\beta, \omega}^{s}} \\
& \times \omega\left(B_{k}\right)^{\frac{1}{q_{2}}-1} \omega\left(B_{k}\right)^{1-\frac{1}{q_{2}}} 2^{(j-k) n \delta\left(1-\frac{1}{q_{2}}\right)} \\
& \leqslant C(k-j) 2^{(j-k) n\left(\frac{\delta}{q_{2}^{\prime}}+\frac{1}{n r}-\frac{1}{r}\right)}\|\Omega\|_{L^{r}\left(S^{n-1}\right)}\left\|f \chi_{E_{j}}\right\|_{L^{q_{1}}\left(\mathbb{R}^{n}, \omega\right)}\|b\|_{\operatorname{Lip}_{\beta, \omega}^{s}} .
\end{aligned}
$$

This inequality together with the estimate of $U_{1}^{\prime}$ deduces that

$$
\left\|[b, T] f_{j} \chi_{k}\right\|_{L^{q_{2}}\left(\mathbb{R}^{n}, \omega^{1-q_{2}}\right)} \leqslant C(k-j) 2^{(j-k) n\left(\min \left\{\frac{1}{q_{1}^{\prime}}, \frac{\delta}{q_{2}^{\prime}}\right\}+\frac{1}{n r}-\frac{1}{r}\right)}\left\|f \chi_{E_{j}}\right\|_{L^{q_{1}}\left(\mathbb{R}^{n}, \omega\right)} .
$$

Therefore, applying Lemma 2.6 and (ii) of Lemma 2.5, we obtain that

$$
\begin{aligned}
& I_{1} \leqslant C \omega(B)^{-p \lambda} \sum_{k=-\infty}^{0} \omega\left(E_{k}\right)^{\alpha p}\left(\sum_{j=-\infty}^{k-2}(k-j) 2^{(j-k) n\left(\min \left\{\frac{1}{q_{1}^{\prime}}, \frac{\delta}{q_{2}^{\prime}}\right\}+\frac{1}{n r}-\frac{1}{r}\right)}\left\|f \chi_{E_{j}}\right\|_{L^{q_{1}}\left(\mathbb{R}^{n}, \omega\right)}\right)^{p} \\
& \leqslant \mathrm{C} \omega(\mathrm{B})^{-p \lambda} \sum_{k=-\infty}^{0} \omega\left(E_{k}\right)^{\alpha p}\left(\sum_{j=-\infty}^{k-2}(k-j) 2^{(j-k) n\left(\min \left\{\frac{1}{q_{1}^{\prime}}, \frac{\delta}{q_{2}^{\prime}}\right\}+\frac{1}{n r}-\frac{1}{r}\right)} \omega\left(E_{j}\right)^{-\alpha+\lambda}\right. \\
& \left.\times\left(\omega\left(E_{j}\right)^{-\lambda p+\alpha p}\left\|f \chi_{E_{j}}\right\|_{L^{q_{1}}\left(\mathbb{R}^{n}, \omega\right)}^{p}\right)^{\frac{1}{p}}\right)^{p} \\
& \leqslant C\|f\|_{L_{p, q_{1}}^{\alpha, \lambda}\left(\mathbb{R}^{n}, \omega, \omega\right)}^{p} \omega(B)^{-p \lambda} \sum_{k=-\infty}^{0} \omega\left(E_{k}\right)^{\alpha p} \\
& \times\left\{\begin{array}{l}
\left(\sum_{j=-\infty}^{k-2}(k-j) 2^{(j-k) n\left(\min \left\{\frac{1}{q_{1}^{\prime}} \frac{\delta}{q_{2}^{\prime}}\right\}+\frac{1}{n r}-\frac{1}{r}\right)} 2^{n(j-k)(\lambda-\alpha)} \omega\left(E_{k}\right)^{\lambda-\alpha}\right)^{p}, \quad \alpha \geqslant \lambda \\
\left(\sum_{j=-\infty}^{k-2}(k-j) 2^{(j-k) n\left(\min \left\{\frac{1}{q_{1}^{\prime}}, \frac{\delta}{q_{2}^{\prime}}\right\}+\frac{1}{n r}-\frac{1}{r}\right)} 2^{n(j-k) \delta(\lambda-\alpha)} \omega\left(E_{k}\right)^{\lambda-\alpha}\right)^{p}, \quad \alpha<\lambda
\end{array}\right. \\
& \leqslant C\|f\|_{L_{p, q_{1}}^{\alpha, \lambda}\left(\mathbb{R}^{n}, \omega, \omega\right)}^{p} \omega(B)^{-p \lambda} \sum_{k=-\infty}^{0} \omega\left(E_{k}\right)^{\lambda p} \\
& \times\left\{\begin{array}{l}
\left(\sum_{j=-\infty}^{k-2}(k-j) 2^{(j-k) n\left(\min \left\{\frac{1}{q_{1}^{\prime}}, \frac{\delta}{q_{2}}\right\}+\frac{1}{n r}-\frac{1}{r}+\lambda-\alpha\right)}\right)^{p}, \quad \alpha \geqslant \lambda \\
\left(\sum_{j=-\infty}^{k-2}(k-j) 2^{(j-k) n\left(\min \left\{\frac{1}{q_{1}^{\prime}}, \frac{\delta}{q_{2}^{2}}\right\}+\frac{1}{n r}-\frac{1}{r}+\lambda \delta-\alpha \delta\right)}\right)^{p}, \quad \alpha<\lambda
\end{array}\right. \\
& \leqslant C\|f\|_{L_{p, q_{1}}^{\alpha, \lambda}\left(\mathbb{R}^{n}, \omega, \omega\right)^{\prime}}^{p}
\end{aligned}
$$

where we require either $\lambda \leqslant \alpha<\min \left\{\frac{1}{\mathrm{q}_{1}^{\prime}}, \frac{\delta}{\mathrm{q}_{2}^{\prime}}\right\}+\frac{1}{n r}-\frac{1}{r}+\lambda$ or $\alpha<\lambda$.

We finally estimate $I_{3}$. When $x \in E_{k}, y \in E_{j}, j \geqslant k+2$, it is obvious to see that $|x-y| \sim\left|x-x_{0}\right| \sim 2^{j} d$. If $r>\max \left\{q_{2}, \frac{(n-1) q_{1}}{n \delta}\right\}$, we obtain, by Minkowski's inequality that

$$
\left\|[b, T] f_{j} \chi_{k}\right\|_{L^{q_{2}}\left(\mathbb{R}^{n}, \omega^{\left.1-q_{2}\right)}\right.} \leqslant C 2^{-j n}\left(\int_{E_{k}}\left(\int_{E_{j}}|\Omega(x-y)\|b(x)-b(y)\| f(y)| d y\right)^{q_{2}} \omega^{1-q_{2}}(x) d x\right)^{\frac{1}{q_{2}}}
$$




$$
\begin{aligned}
\leqslant & C 2^{-j n} \int_{E_{j}}\left(\int_{E_{k}}|\Omega(x-y)|^{q_{2}}\left|b(x)-b_{B_{k}}\right| q^{q_{2}} w^{1-q_{2}}(x) d x\right)^{\frac{1}{q_{2}}}|f(y)| d y \\
& +C 2^{-j n} \int_{E_{j}}\left(\int_{E_{k}}|\Omega(x-y)|^{q_{2}} \omega^{1-q_{2}}(x) d x\right)^{\frac{1}{q_{2}}}\left|b_{B_{k}}-b(y)\right||f(y)| d y \\
:= & C\left(V_{1}+V_{2}\right) .
\end{aligned}
$$

Choose $s>\max \left\{\frac{q_{2} r}{r-q_{2}}, q_{1}^{\prime}\right\}$. By the same line to estimate $U_{1}$ and $U_{2}$, we get that

$$
\begin{aligned}
& V_{1} \leqslant C 2^{-j n} \int_{E_{j}}\left(\int_{E_{k}}|\Omega(x-y)|^{r} d x\right)^{\frac{1}{r}}\left(\int_{B_{k}}\left|b(x)-b_{B_{k}}\right|^{s} \omega^{1-s}(x) d x\right)^{\frac{1}{s}}
\end{aligned}
$$

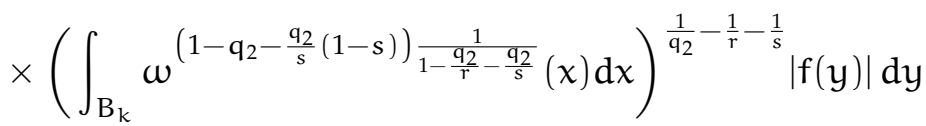

$$
\begin{aligned}
& \leqslant C 2^{-j n}\|\Omega\|_{L^{r}\left(S^{n-1}\right)} 2^{\frac{j(n-1)}{r}+\frac{k}{r}}\|b\|_{L_{\beta, \omega}^{s} s} \omega\left(B_{k}\right)^{\frac{\beta}{n}+\frac{1}{s}} \omega\left(B_{k}\right)^{\frac{1}{q_{2}}-\frac{1}{s}}\left|B_{k}\right|^{-\frac{1}{r}}\left\|f \chi_{E_{j}}\right\|_{L^{q_{1}}\left(\mathbb{R}^{n}, \omega\right)}\left|B_{j}\right| \omega\left(B_{j}\right)^{-\frac{1}{q_{1}}}
\end{aligned}
$$

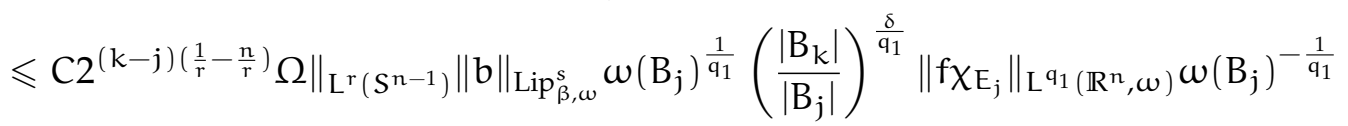

$$
\begin{aligned}
& \leqslant C 2^{(k-j) n\left(\frac{\delta}{q_{1}}+\frac{1}{n r}-\frac{1}{r}\right)} \Omega\left\|_{L^{r}\left(S^{n-1}\right)}\right\| b\left\|_{\operatorname{Lip}_{\beta, \omega}^{s}}\right\| f \chi_{E_{j}} \|_{L^{q_{1}}\left(\mathbb{R}^{n}, w\right)}
\end{aligned}
$$

and

$$
\begin{aligned}
& V_{2} \leqslant C 2^{-j n} \int_{E_{j}}\left(\int_{E_{k}}|\Omega(x-y)|^{r} d x\right)^{\frac{1}{r}}\left(\int_{B_{k}} \omega^{\left(1-q_{2}\right)\left(\frac{r}{q_{2}}\right)^{\prime}}(x) d x\right)^{\frac{1}{q_{2}}-\frac{1}{r}}\left|b_{B_{k}}-b(y) \| f(y)\right| d y \\
& \leqslant C 2^{-j n}\|\Omega\|_{L^{r}\left(S^{n-1}\right)} 2^{\frac{j(n-1)}{r}+\frac{k}{r}} \omega\left(B_{k}\right)^{\frac{1}{q_{2}}-1}\left|B_{k}\right|^{1-\frac{1}{r}}\left\|f \chi_{E_{j}}\right\|_{L^{q_{1}}\left(\mathbb{R}^{n}, \omega\right)} \\
& \times(j-k) \omega\left(B_{j}\right)^{\frac{\beta}{n}+\frac{1}{s}}\|b\|_{\operatorname{Lip}_{\beta, \omega}^{s}} \omega\left(B_{j}\right)^{1-\frac{1}{q_{1}}-\frac{1}{s}} \\
& \leqslant C(j-k) 2^{(k-j) n\left(1-\frac{1}{r}+\frac{1}{n r}\right)}\|\Omega\|_{L^{r}\left(S^{n-1}\right)}\left\|f \chi_{E_{j}}\right\|_{L^{q_{1}}\left(\mathbb{R}^{n}, \omega\right)}\|b\|_{L_{\beta, \omega}^{s}} \omega\left(B_{k}\right)^{\frac{1}{q_{2}}-1} \omega\left(B_{j}\right)^{1-\frac{1}{q_{2}}} \\
& \leqslant C(j-k) 2^{(k-j) n\left(1-\frac{1}{r}+\frac{1}{n r}\right)}\|\Omega\|_{L^{r}\left(S^{n-1}\right)}\left\|f \chi_{E_{j}}\right\|_{L^{q_{1}}\left(\mathbb{R}^{n}, \omega\right)}\|b\|_{\operatorname{Lip}_{\beta, \omega}^{s} \omega\left(B_{k}\right)^{\frac{1}{q_{2}}-1}} \\
& \times \omega\left(B_{k}\right)^{1-\frac{1}{q_{2}}} 2^{(j-k) n\left(1-\frac{1}{q_{2}}\right)} \\
& \leqslant C(j-k) 2^{(k-j) n\left(\frac{1}{q_{2}}-\frac{1}{r}+\frac{1}{n r}\right)}\|\Omega\|_{L^{r}\left(S^{n-1}\right)}\left\|f \chi_{E_{j}}\right\|_{L^{q_{1}}\left(\mathbb{R}^{n}, w\right)}\|b\|_{\operatorname{Lip}_{\beta, \omega}^{s}},
\end{aligned}
$$

which along with estimate of $V_{1}$ reads that

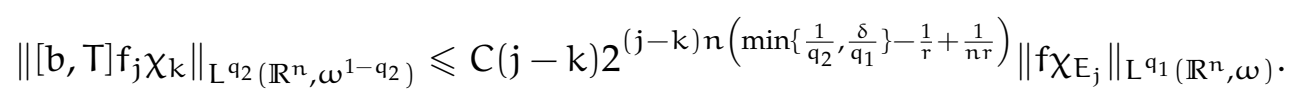

With the help of Lemma 2.6 and (i) of Lemma 2.5, we now obtain that

$$
\begin{aligned}
I_{3} \leqslant & C \omega(B)^{-p \lambda} \sum_{k=-\infty}^{0} \omega\left(E_{k}\right)^{\alpha p}\left(\sum_{j=k+2}^{\infty}(j-k) 2^{(k-j) n\left(\min \left\{\frac{1}{q_{2}}, \frac{\delta}{q_{1}}\right\}-\frac{1}{r}+\frac{1}{n r}\right)}\left\|f \chi_{E_{j}}\right\|_{L^{q_{1}}\left(\mathbb{R}^{n}, \omega\right)}\right)^{p} \\
\leqslant & C \omega(B)^{-p \lambda} \sum_{k=-\infty}^{0} \omega\left(E_{k}\right)^{\alpha p}\left(\sum_{j=k+2}^{\infty}(j-k) 2^{(k-j) n\left(\min \left\{\frac{1}{q_{2}}, \frac{\delta}{q_{1}}\right\}-\frac{1}{r}+\frac{1}{n r}\right)} \omega\left(E_{j}\right)^{-\alpha+\lambda}\right. \\
& \left.\times\left(\omega\left(E_{j}\right)^{-\lambda p+\alpha p}\left\|f \chi_{E_{j}}\right\|_{L^{q_{1}}\left(\mathbb{R}^{n}, \omega\right)}^{p}\right)^{\frac{1}{p}}\right)^{p} \\
\leqslant & C\|f\|_{L_{p, q_{1}}^{\alpha, \lambda}\left(\mathbb{R}^{n}, \omega, \omega\right)}^{p} \omega(B)^{-p \lambda} \sum_{k=-\infty}^{0} \omega\left(E_{k}\right)^{\alpha p}
\end{aligned}
$$




$$
\begin{aligned}
& \times\left\{\begin{array}{l}
\left(\sum_{j=k+2}^{\infty}(j-k) 2^{(k-j) n\left(\min \left\{\frac{1}{q_{2}}, \frac{\delta}{q_{1}}\right\}-\frac{1}{r}+\frac{1}{n r}\right)} 2^{n(j-k) \delta(\lambda-\alpha)} \omega\left(E_{k}\right)^{\lambda-\alpha}\right)^{p}, \alpha \geqslant \lambda \\
\left(\sum_{j=k+2}^{\infty}(j-k) 2^{(k-j) n\left(\min \left\{\frac{1}{q_{2}}, \frac{\delta}{q_{1}}\right\}-\frac{1}{r}+\frac{1}{n r}\right)} 2^{n(j-k)(\lambda-\alpha)} \omega\left(E_{k}\right)^{\lambda-\alpha}\right)^{p}, \quad \alpha<\lambda
\end{array}\right. \\
& \leqslant C\|f\|_{L_{p, q_{1}}^{\alpha, \lambda}\left(\mathbb{R}^{n}, \omega, \omega\right)}^{p} \omega(B)^{-p \lambda} \sum_{k=-\infty}^{0} \omega\left(E_{k}\right)^{\lambda p} \\
& \quad \times\left\{\begin{array}{l}
\left(\sum_{j=k+2}^{\infty}(j-k) 2^{(k-j) n\left(\min \left\{\frac{1}{q_{2}}, \frac{\delta}{q_{1}}\right\}-\frac{1}{r}+\frac{1}{n r}-\lambda \delta+\alpha \delta\right)}\right)^{p}, \quad \alpha \geqslant \lambda \\
\left(\sum_{j=k+2}^{\infty}(j-k) 2^{(k-j) n\left(\min \left\{\frac{1}{q_{2}}, \frac{\delta}{q_{1}}\right\}-\frac{1}{r}+\frac{1}{n r}-\lambda+\alpha\right)}\right)^{p}, \quad \alpha<\lambda
\end{array}\right. \\
& \leqslant C\|f\|_{L_{p, q_{1}}^{\alpha, \lambda}\left(\mathbb{R}^{n}, \omega, \omega\right)^{\prime}}^{p}
\end{aligned}
$$

where we need either $\alpha \geqslant \lambda$ or $\lambda+\frac{1}{r}-\frac{1}{n r}-\min \left\{\frac{1}{q_{2}}, \frac{\delta}{q_{1}}\right\}<\alpha<\lambda$.

If $r>\max \left\{q_{1}^{\prime}, \frac{q_{2}^{\prime}(n-1)}{n \delta}\right\}$, we write

$$
\begin{aligned}
\left\|[b, T] f_{j} \chi_{k}\right\|_{L^{q_{2}}\left(\mathbb{R}^{n}, w^{1-q_{2}}\right)} & =C 2^{-j n}\left(\int_{E_{k}}\left(\int_{E_{j}}|\Omega(x-y)\|b(x)-b(y)\| f(y)| d y\right)^{q_{2}} \omega^{1-q_{2}}(x) d x\right)^{\frac{1}{q_{2}}} \\
\leqslant & C 2^{-j n}\left(\int_{E_{k}}\left|b(x)-b_{B_{k}}\right|^{q_{2}}\left(\int_{E_{j}}|\Omega(x-y) \| f(y)| d y\right)^{q_{2}} \omega^{1-q_{2}}(x) d x\right)^{\frac{1}{q_{2}}} \\
& +C 2^{-j n}\left(\int_{E_{k}}\left(\int_{E_{j}}\left|\Omega(x-y)\left\|b_{B_{k}}-b(y)\right\| f(y)\right| d y\right)^{q_{2}} \omega^{1-q_{2}}(x) d x\right)^{\frac{1}{q_{2}}} \\
:= & C\left(V_{1}^{\prime}+V_{2}^{\prime}\right) .
\end{aligned}
$$

By the same methods used to estimate $U_{1}^{\prime}$ and $U_{2}^{\prime}$, we obtain that

$$
\begin{aligned}
& V_{1}^{\prime} \leqslant C 2^{-j n}\left(\int _ { E _ { k } } | b ( x ) - b _ { B _ { k } } | ^ { q _ { 2 } } \left(\left(\int_{E_{j}}|\Omega(x-y)|^{q_{1}^{\prime}} w(y)^{-\frac{q_{1}^{\prime}}{q_{1}}} d y\right)^{\frac{1}{q_{1}^{\prime}}}\right.\right. \\
& \left.\left.\times\left(\int_{E_{j}}|f(y)|^{q_{1}} \omega(y) d y\right)^{\frac{1}{q_{1}}}\right)^{q_{2}} w^{1-q_{2}}(x) d x\right)^{\frac{1}{q_{2}}}
\end{aligned}
$$

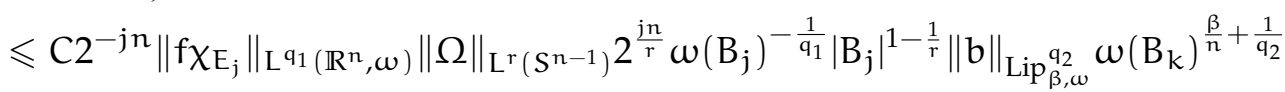

$$
\begin{aligned}
& \leqslant C\left\|f \chi_{E_{j}}\right\|_{L^{q_{1}}\left(\mathbb{R}^{n}, \omega\right)}\|\Omega\|_{L^{r}\left(S^{n-1}\right)} \omega\left(B_{j}\right)^{-\frac{1}{q_{1}}}\|b\|_{L_{\beta_{\beta, \omega}}^{q_{2}} \omega\left(B_{j}\right)^{\frac{1}{q_{1}}}}\left(\frac{\left|B_{k}\right|}{\left|B_{j}\right|}\right)^{\frac{\delta}{q_{1}}} \\
& \leqslant C 2^{(k-j) n \frac{\delta}{q_{1}}}\|\Omega\|_{L^{r}\left(S^{n-1}\right)}\left\|f \chi_{E_{j}}\right\|_{L^{q_{1}\left(\mathbb{R}^{n}, \omega\right)}}\|b\|_{\operatorname{Lip}_{\beta, \omega}^{q_{2}}} \text {, }
\end{aligned}
$$

and

$$
\begin{aligned}
V_{2}^{\prime} & =C 2^{-j n}\left(\int_{E_{k}}\left(\int_{E_{j}}\left|\Omega(x-y)\left\|b_{B_{k}}-b(y)\right\| f(y)\right| d y\right)^{q_{2}} \omega^{1-q_{2}}(x) d x\right)^{\frac{1}{q_{2}}} \\
& \leqslant C 2^{-j n}\left(\int _ { E _ { k } } \left(\int_{E_{j}}|\Omega(x-y)|^{q_{1}^{\prime}}\left|b_{B_{k}}-b(y)\right|^{q_{1}^{\prime}} \omega(y)^{\left.-\frac{q_{1}^{\prime}}{q_{1}} d y\right)^{\frac{q_{2}}{q_{1}^{\prime}}}}\right.\right.
\end{aligned}
$$




$$
\begin{aligned}
& \left.\times\left(\int_{E_{j}}|f(y)|^{q_{1}} \omega(y) d y\right)^{\frac{q_{2}}{q_{1}}} \omega(x)^{1-q_{2}} d x\right)^{\frac{1}{q_{2}}} \\
& \leqslant C(j-k) 2^{-j n} 2^{\frac{j n}{r}}\left\|f \chi_{E_{j}}\right\|_{L^{q_{1}}\left(\mathbb{R}^{n}, \omega\right)}\|\Omega\|_{L^{r}\left(S^{n-1}\right)} \omega\left(B_{j}\right)^{\frac{\beta}{n}+\frac{1}{s}}\|b\|_{L_{\beta, \omega}^{s}}\left|B_{j}\right|^{-\frac{1}{r}} \\
& \times \omega\left(B_{j}\right)^{\frac{1}{q_{1}^{\prime}}-\frac{1}{s}}\left|B_{k}\right| \omega\left(B_{k}\right)^{\frac{1}{q_{2}}-1} \\
& \leqslant C(j-k) 2^{(k-j) n}\|\Omega\|_{L^{r}\left(S^{n-1}\right)}\left\|f \chi_{E_{j}}\right\|_{L^{q_{1}}\left(\mathbb{R}^{n}, \omega\right)}\|b\|_{L_{\beta, \omega}^{s}} \omega\left(B_{k}\right)^{\frac{1}{q_{2}}-1} \omega\left(B_{k}\right)^{1-\frac{1}{q_{2}}} 2^{(j-k) n\left(1-\frac{1}{q_{2}}\right)} \\
& \leqslant C(j-k) 2^{(k-j) n \frac{1}{q_{2}}}\|\Omega\|_{L^{r}\left(S^{n-1}\right)}\left\|f \chi_{E_{j}}\right\|_{L^{q_{1}\left(\mathbb{R}^{n}, \omega\right)}}\|b\|_{\operatorname{Lip}_{\beta, \omega}^{s}} \text {. }
\end{aligned}
$$

These estimates tell that

$$
\left\|[b, T] f_{j} \chi_{k}\right\|_{L^{q_{2}}\left(\mathbb{R}^{n}, w^{1-q_{2}}\right)} \leqslant C(j-k) 2^{(k-j) n \min \left\{\frac{\delta}{q_{1}}, \frac{1}{q_{2}}\right\}}\left\|f_{E_{j}}\right\|_{L^{q_{1}}\left(\mathbb{R}^{n}, w\right) .} .
$$

From Lemma 2.6 and (i) of Lemma 2.5, it follows that

$$
\begin{aligned}
& I_{3} \leqslant C \omega(B)^{-p} \lambda \sum_{k=-\infty}^{0} \omega\left(E_{k}\right)^{\alpha p}\left(\sum_{j=k+2}^{\infty}(j-k) 2^{(k-j) n \min \left\{\frac{\delta}{q_{1}}, \frac{1}{q_{2}}\right\}}\left\|f \chi_{E_{j}}\right\|_{L^{q_{1}}\left(\mathbb{R}^{n}, \omega\right)}\right)^{p} \\
& \leqslant \mathrm{C} \omega(\mathrm{B})^{-\mathrm{p} \lambda} \sum_{k=-\infty}^{0} \omega\left(\mathrm{E}_{k}\right)^{\alpha p}\left(\sum_{j=k+2}^{\infty}(j-k) 2^{(k-j) n \min \left\{\frac{\delta}{q_{1}}, \frac{1}{q_{2}}\right\}} \omega\left(E_{j}\right)^{-\alpha+\lambda}\right. \\
& \left.\times\left(w\left(E_{j}\right)^{-\lambda p+\alpha p}\left\|f \chi_{E_{j}}\right\|_{L^{q_{1}}\left(\mathbb{R}^{n}, w\right)}^{p}\right)^{\frac{1}{p}}\right)^{p} \\
& \leqslant C\|f\|_{L_{p, q_{1}}^{\alpha, \lambda}\left(\mathbb{R}^{n}, \omega, \omega\right)}^{p} \omega(B)^{-p \lambda} \sum_{k=-\infty}^{0} \omega\left(E_{k}\right)^{\alpha p} \\
& \times\left\{\begin{array}{l}
\left(\sum_{j=k+2}^{\infty}(j-k) 2^{(k-j) n \min \left\{\frac{\delta}{q_{1}}, \frac{1}{q_{2}}\right\}} 2^{n(j-k) \delta(\lambda-\alpha)} \omega\left(E_{k}\right)^{\lambda-\alpha}\right)^{p}, \quad \alpha \geqslant \lambda \\
\left(\sum_{j=k+2}^{\infty}(j-k) 2^{(k-j) n \min \left\{\frac{\delta}{q_{1}}, \frac{1}{q_{2}}\right\}} 2^{n(j-k)(\lambda-\alpha)} \omega\left(E_{k}\right)^{\lambda-\alpha}\right)^{p}, \quad \alpha<\lambda
\end{array}\right. \\
& \leqslant C\|f\|_{L_{p, q_{1}}^{\alpha, \lambda}\left(\mathbb{R}^{n}, \omega, \omega\right)}^{p} \omega(B)^{-p \lambda} \sum_{k=-\infty}^{0} \omega\left(E_{k}\right)^{\lambda p} \\
& \times\left\{\begin{array}{l}
\left(\sum_{j=k+2}^{\infty}(j-k) 2^{(k-j) n\left(\min \left\{\frac{\delta}{q_{1}}, \frac{1}{q_{2}}\right\}-\lambda \delta+\delta \alpha\right)}\right)^{p}, \quad \alpha \geqslant \lambda \\
\left(\sum_{j=k+2}^{\infty}(j-k) 2^{(k-j) n\left(\min \left\{\frac{\delta}{q_{1}}, \frac{1}{q_{2}}\right\}-\lambda+\alpha\right)}\right)^{p}, \quad \alpha<\lambda
\end{array}\right. \\
& \leqslant C\|f\|_{L_{p, q_{1}}^{\alpha, \lambda}\left(\mathbb{R}^{n}, \omega, w\right)^{\prime}}^{p}
\end{aligned}
$$

where we demand either $\alpha \geqslant \lambda$ or $\lambda-\min \left\{\frac{\delta}{q_{1}}, \frac{1}{q_{2}}\right\}<\alpha<\lambda$.

\section{Weighted boundedness for the commutators of fractional type operator}

In this section, we will prove Theorem 1.8. We skip the proof for Theorem 1.10, since it is the same. Note that if $p_{1} \leqslant p_{2}$, then

$$
\mathrm{L}_{\mathbf{p}_{1}, \mathbf{q}_{2}}^{\alpha, \lambda}\left(\omega, \omega^{1-\mathrm{q}_{2}}\right) \subset \mathrm{L}_{\mathbf{p}_{2}, \mathbf{q}_{2}}^{\alpha, \lambda}\left(\omega, \omega^{1-q_{2}}\right)
$$


So we need only to prove Theorem 1.8 for the case $p_{1}=p_{2}=p$.

For a given ball $B=B\left(x_{0}, d\right)$, let $E_{j}=B\left(x_{0}, 2^{j} d\right) \backslash B\left(x_{0}, 2^{j-1} d\right)$. We decompose $f$ as

$$
f(y)=\sum_{j=-\infty}^{\infty} f(y) \chi_{E_{j}}(y)=\sum_{j=-\infty}^{\infty} f_{j}(y) .
$$

Then we can write

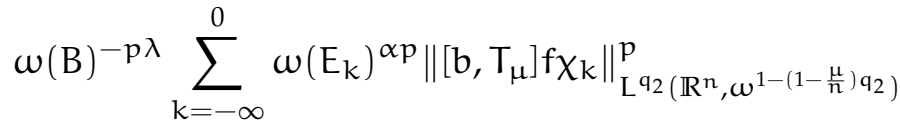

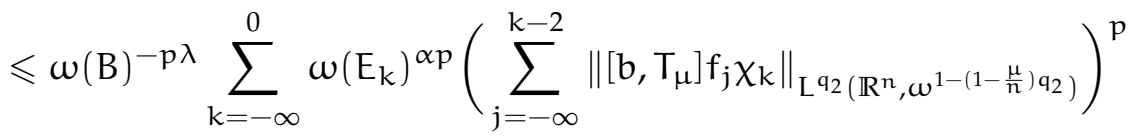

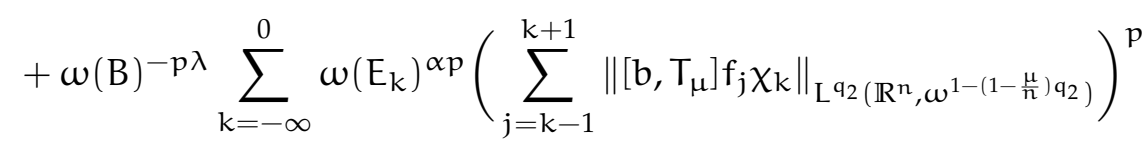

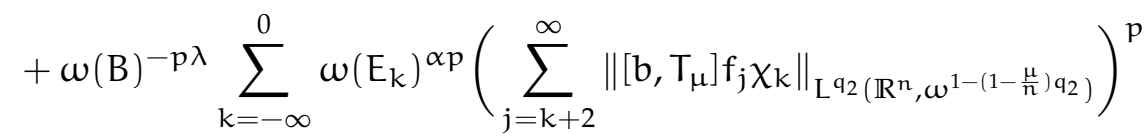

$$
\begin{aligned}
& =: \mathrm{I}_{1}+\mathrm{I}_{2}+\mathrm{I}_{3} \text {. }
\end{aligned}
$$

The known $\left(\mathrm{L}^{\mathrm{q}_{1}}\left(\mathbb{R}^{\mathrm{n}}, \omega\right), \mathrm{L}^{\mathrm{q}_{2}}\left(\mathbb{R}^{\mathrm{n}}, \omega^{1-\left(1-\frac{\mu}{n}\right) \mathrm{q}_{2}}\right)\right)$-boundedness of commutator $\left[b, T_{\mu}\right]$ gives that

$$
I_{2} \leqslant \omega(B)^{-p \lambda} \sum_{k=-\infty}^{0} \omega\left(E_{k}\right)^{\alpha p}\left(\sum_{j=k-1}^{k+1}\left\|f_{j} \chi_{k}\right\|_{L^{q_{1}}\left(\mathbb{R}^{n}, \omega\right)}\right)^{p} \leqslant C\|f\|_{L_{p, q_{1}}^{\alpha, \lambda}\left(\mathbb{R}^{n}, \omega, \omega\right)}^{p} .
$$

Now we bound $I_{1}$. When $x \in E_{k}, y \in E_{j}, j \leqslant k-2$, then it is easy to check $|x-y| \sim\left|x-x_{0}\right| \sim 2^{k} d$. If $r>q_{2}$, then the Minkowski inequality implies

$$
\begin{aligned}
\left\|\left[b, T_{\mu}\right] f_{j} \chi_{k}\right\|_{L^{q_{2}}\left(\mathbb{R}^{n}, w^{1-\left(1-\frac{\mu}{n}\right) q_{2}}\right.} \\
\leqslant C 2^{-k n+k \mu}\left(\int_{E_{k}}\left(\int_{E_{j}}|\Omega(x-y) \| b(x)-b(y)||f(y)| d y\right)^{q_{2}} w^{1-\left(1-\frac{\mu}{n}\right) q_{2}}(x) d x\right)^{\frac{1}{q_{2}}} \\
\leqslant C 2^{-k n+k \mu} \int_{E_{j}}\left(\int_{E_{k}}|\Omega(x-y)|^{q_{2}}\left|b(x)-b_{B_{k}}\right|{ }^{q_{2}} w^{1-\left(1-\frac{\mu}{n}\right) q_{2}}(x) d x\right)^{\frac{1}{q_{2}}}|f(y)| d y \\
\quad+C 2^{-k n+k \mu} \int_{E_{j}}\left(\int_{E_{k}}|\Omega(x-y)|^{q_{2}} w^{1-\left(1-\frac{\mu}{n}\right) q_{2}}(x) d x\right)^{\frac{1}{q_{2}}}\left|b_{B_{k}}-b(y) \| f(y)\right| d y \\
:=C\left(U_{1}+U_{2}\right) .
\end{aligned}
$$

Choose $s>\max \left\{\frac{q_{2} r}{r-q_{2}}, q_{1}^{\prime}\right\}$. By Hölder's inequality, (ii) of Lemma 2.2, the reverse Hölder inequality and Lemma 2.4, we obtain that

$$
\begin{aligned}
& \mathrm{U}_{1} \leqslant \mathrm{C} 2^{-k n+k \mu} \int_{\mathrm{E}_{j}}\left(\int_{\mathrm{E}_{k}}|\Omega(x-y)|^{\mathrm{r}} \mathrm{d} x\right)^{\frac{1}{\mathrm{r}}}\left(\int_{\mathrm{B}_{k}}\left|\mathrm{~b}(x)-\mathrm{b}_{\mathrm{B}_{k}}\right|^{s} \omega^{1-s}(x) d x\right)^{\frac{1}{s}}
\end{aligned}
$$

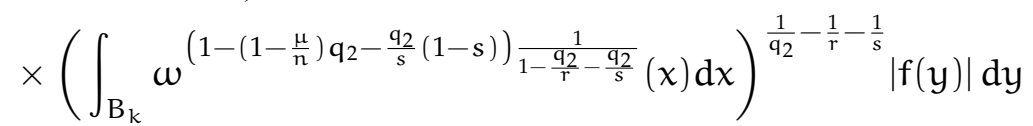

$$
\begin{aligned}
& \leqslant C 2^{-k n+k \mu}\|\Omega\|_{L^{r}\left(S^{n-1}\right)} 2^{\frac{k n}{r}}\|b\|_{L_{\beta, \omega}^{s}}^{s} \omega\left(B_{k}\right)^{\frac{\beta}{n}+\frac{1}{s}} \omega\left(B_{k}\right)^{\frac{1}{q_{2}}-\frac{1}{s}+\frac{\mu}{n}}\left|B_{k}\right|^{-\frac{1}{r}-\frac{\mu}{n}} \int_{E_{j}}|f(y)| d y
\end{aligned}
$$




$$
\begin{aligned}
& \leqslant C 2^{-k n}\|\Omega\|_{L^{r}\left(S^{n-1}\right)}\|b\|_{\operatorname{Lip}_{\beta, \omega}^{s}} \omega\left(B_{k}\right)^{\frac{1}{q_{1}}}\left\|f \chi_{E_{j}}\right\|_{L^{q_{1}}\left(\mathbb{R}^{n}, \omega\right)}\left|B_{j}\right| \omega\left(B_{j}\right)^{-\frac{1}{q_{1}}} \\
& \leqslant C 2^{(j-k) n}\|\Omega\|_{L^{r}\left(S^{n-1}\right)}\|b\|_{\operatorname{Lip}_{\beta, \omega}^{s}} \omega\left(B_{j}\right)^{\frac{1}{q_{1}}}\left(\frac{\left|B_{k}\right|}{\left|B_{j}\right|}\right)^{\frac{1}{q_{1}}}\left\|f \chi_{E_{j}}\right\| \|_{L_{1}^{q_{1}}\left(\mathbb{R}^{n}, \omega\right)} \omega\left(B_{j}\right)^{-\frac{1}{q_{1}}} \\
& \leqslant C 2^{(j-k) \frac{n}{q_{1}^{\prime}}}\|\Omega\|_{L^{r}\left(S^{n-1}\right)}\|b\|_{\operatorname{Lip}_{\beta, \omega}^{s}}\left\|f \chi_{E_{j}}\right\|_{L^{q_{1}}\left(\mathbb{R}^{n}, \omega\right)} .
\end{aligned}
$$

Thanks to Hölder's inequality, reverse Hölder inequality and (ii) of Lemma 2.2 again, we have that

$$
\begin{aligned}
& \mathrm{U}_{2} \leqslant C 2^{-k n+k \mu} \int_{E_{j}}\left(\int_{E_{k}}|\Omega(x-y)|^{r} d x\right)^{\frac{1}{r}}\left(\int_{B_{k}} w^{\left(1-\left(1-\frac{\mu}{n}\right) q_{2}\right)\left(\frac{r}{q_{2}}\right)^{\prime}}(x) d x\right)^{\frac{1}{q_{2}}-\frac{1}{r}}\left|b_{B_{k}}-b(y)\right||f(y)| d y \\
& \leqslant C 2^{-k n+k \mu}\|\Omega\|_{L^{r}\left(S^{n-1}\right)} 2^{\frac{k n}{r}} \omega\left(B_{k}\right)^{\frac{1}{q_{2}}-1+\frac{\mu}{n}}\left|B_{k}\right|^{1-\frac{\mu}{n}-\frac{1}{r}}\left(\int_{E_{j}}|f(y)|^{q_{1}} \omega d y\right)^{\frac{1}{q_{1}}} \\
& \times\left(\int_{B_{j}}\left|b_{B_{k}}-b(y)\right|^{s} w(y)^{1-s} d y\right)^{\frac{1}{s}}\left(\int_{B_{j}} w(y)^{\left(\frac{s-1}{s}-\frac{1}{q_{1}}\right) \frac{1}{1-\frac{1}{q_{1}}-\frac{1}{s}}} d y\right)^{1-\frac{1}{q_{1}}-\frac{1}{s}} \\
& \leqslant C\|\Omega\|_{L^{r}\left(S^{n-1}\right)} \omega\left(B_{k}\right)^{\frac{1}{q_{2}}-1+\frac{\mu}{n}}\left\|f \chi_{E_{j}}\right\|_{L^{q_{1}\left(\mathbb{R}^{n}, \omega\right)}}(k-j) \omega\left(B_{j}\right)^{\frac{\beta}{n}+\frac{1}{s}}\|b\|_{\operatorname{Lip}_{\beta, \omega}^{s}} \omega\left(B_{j}\right)^{1-\frac{1}{q_{1}}-\frac{1}{s}} \\
& \leqslant C(k-j)\|\Omega\|_{L^{r}\left(S^{n-1}\right)}\left\|f \chi_{E_{j}}\right\|_{L^{q_{1}}\left(\mathbb{R}^{n}, \omega\right)}\|b\|_{L_{\beta, \omega}^{s}} \omega\left(B_{k}\right)^{\frac{1}{q_{2}}-1+\frac{\mu}{n}} \omega\left(B_{j}\right)^{\frac{\beta}{n}+1-\frac{1}{q_{1}}}
\end{aligned}
$$

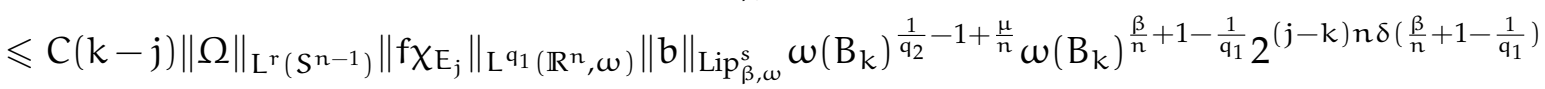

$$
\begin{aligned}
& \leqslant C(k-j) 2^{(j-k) n \delta\left(\frac{\beta}{n}+\frac{1}{q_{1}^{\prime}}\right)}\|\Omega\|_{L^{r}\left(S^{n-1}\right)}\|b\|_{L_{\beta, \omega}^{s} s}\left\|f \chi_{E_{j}}\right\|_{L^{q_{1}}\left(\mathbb{R}^{n}, \omega\right)} \text {. }
\end{aligned}
$$

Combining the estimates of $\mathrm{U}_{2}$ and $\mathrm{U}_{1}$, we now have that

$$
\left\|\left[b, T_{\mu}\right] f_{j} \chi_{k}\right\|_{L^{q_{2}}\left(\mathbb{R}^{n}, \omega^{\left.1-\left(1-\frac{\mu}{n}\right) q_{2}\right)}\right.} \leqslant C(k-j) 2^{(j-k) n \min \left\{\frac{1}{q_{1}^{\prime}} \delta\left(\frac{\beta}{n}+\frac{1}{q_{1}^{\prime}}\right)\right\}}\left\|f \chi_{E_{j}}\right\|_{L^{q_{1}}\left(\mathbb{R}^{n}, \omega\right)} .
$$

The above inequalities together with Lemma 2.6 and (ii) of Lemma 2.5 yield

$$
\begin{aligned}
& I_{1} \leqslant C \omega(B)^{-p \lambda} \sum_{k=-\infty}^{0} \omega\left(E_{k}\right)^{\alpha p}\left(\sum_{j=-\infty}^{k-2}(k-j) 2^{(j-k) n \min \left\{\frac{1}{q_{1}^{\prime}}, \delta\left(\frac{\beta}{n}+\frac{1}{q_{1}^{\prime}}\right)\right\}}\left\|f \chi_{E_{j}}\right\|_{L^{q_{1}}\left(\mathbb{R}^{n}, \omega\right)}\right)^{p} \\
& \leqslant C \omega(B)^{-p \lambda} \sum_{k=-\infty}^{0} \omega\left(E_{k}\right)^{\alpha p}\left(\sum_{j=-\infty}^{k-2}(k-j) 2^{(j-k) n \min \left\{\frac{1}{q_{1}^{\prime}}, \delta\left(\frac{\beta}{n}+\frac{1}{q_{1}^{\prime}}\right)\right\}} \omega\left(E_{j}\right)^{-\alpha+\lambda}\right. \\
&\left.\times\left(\omega\left(E_{j}\right)^{-\lambda p+\alpha p}\left\|f \chi_{E_{j}}\right\|_{L^{q_{1}}\left(\mathbb{R}^{n}, \omega\right)}^{p}\right)^{\frac{1}{p}}\right)^{p} \\
& \leqslant C\|f\|_{L_{p, q}^{\alpha, q}\left(\mathbb{R}^{n}, \omega, \omega\right)}^{p} \omega(B)^{-p \lambda} \sum_{k=-\infty}^{0} \omega\left(E_{k}\right)^{\alpha p} \\
& \times\left\{\sum_{j=-\infty}^{k-2}(k-j) 2^{(j-k) n \min \left\{\frac{1}{q_{1}^{\prime}}, \delta\left(\frac{\beta}{n}+\frac{1}{q_{1}^{\prime}}\right)\right\}} 2^{n(j-k)(\lambda-\alpha)} \omega\left(E_{k}\right)^{\lambda-\alpha}\right)^{p}, \quad \alpha \geqslant \lambda \\
&\left(\sum_{j=-\infty}^{k-2}(k-j) 2^{(j-k) n \min \left\{\frac{1}{q_{1}^{\prime}}, \delta\left(\frac{\beta}{n}+\frac{1}{q_{1}^{\prime}}\right)\right\}} 2^{n(j-k) \delta(\lambda-\alpha)} \omega\left(E_{k}\right)^{\lambda-\alpha}\right)^{p}, \quad \alpha<\lambda \\
& \leqslant
\end{aligned}
$$




$$
\begin{aligned}
& \times\left\{\begin{array}{l}
\left(\sum_{j=-\infty}^{k-2}(k-j) 2^{(j-k) n\left(\min \left\{\frac{1}{q_{1}^{\prime}}, \delta\left(\frac{\beta}{n}+\frac{1}{q_{1}^{\prime}}\right)\right\}+\lambda-\alpha\right)}\right)^{p}, \quad \alpha \geqslant \lambda \\
\left(\sum_{j=-\infty}^{k-2}(k-j) 2^{(j-k) n\left(\min \left\{\frac{1}{q_{1}^{\prime}}, \delta\left(\frac{\beta}{n}+\frac{1}{q_{1}^{\prime}}\right)\right\}+\lambda \delta-\alpha \delta\right)}\right)^{p}, \quad \alpha<\lambda
\end{array}\right. \\
& \leqslant C\|f\|_{L_{p, q_{1}}^{\alpha, \lambda}\left(\mathbb{R}^{n}, \omega, \omega\right)^{\prime}}^{p}
\end{aligned}
$$

where we require either $\lambda \leqslant \alpha<\lambda+\min \left\{\frac{1}{\mathrm{q}_{1}^{\prime}}, \delta\left(\frac{\beta}{\mathrm{n}}+\frac{1}{\mathrm{q}_{1}^{\prime}}\right)\right\}$ or $\alpha<\lambda$.

If $r>\max \left\{q_{1}^{\prime}, \frac{q_{1}^{\prime}(n-1)}{\left(q_{1}^{\prime} \beta+n\right) \delta}\right\}$, we write that

$$
\begin{aligned}
& \left\|\left[b, T_{\mu}\right] f_{j} \chi_{k}\right\|_{L_{2} q_{2}\left(\mathbb{R}^{n}, w^{\left.1-\left(1-\frac{\mu}{n}\right) q_{2}\right)}\right.} \\
& \leqslant C 2^{-k n+k \mu}\left(\int_{E_{k}}\left(\int_{E_{j}}|\Omega(x-y)\|b(x)-b(y)\| f(y)| d y\right)^{q_{2}} \omega^{1-\left(1-\frac{\mu}{n}\right) q_{2}}(x) d x\right)^{\frac{1}{q_{2}}} \\
& \leqslant C 2^{-k n+k \mu}\left(\int_{E_{k}}\left|b(x)-b_{B_{k}}\right|^{q_{2}}\left(\int_{E_{j}}|\Omega(x-y) \| f(y)| d y\right)^{q_{2}} w^{1-\left(1-\frac{\mu}{n}\right) q_{2}}(x) d x\right)^{\frac{1}{q_{2}}} \\
& +C 2^{-k n+k \mu}\left(\int_{E_{k}}\left(\int_{E_{j}}\left|\Omega(x-y)\left\|b_{B_{k}}-b(y)\right\| f(y)\right| d y\right)^{q_{2}} \omega^{1-\left(1-\frac{\mu}{n}\right) q_{2}}(x) d x\right)^{\frac{1}{q_{2}}} \\
& :=\mathrm{C}\left(\mathrm{U}_{1}^{\prime}+\mathrm{U}_{2}^{\prime}\right) \text {. }
\end{aligned}
$$

Choose $s>\max \left\{q_{2}, \frac{q_{1}^{\prime} r}{r-q_{1}^{\prime}}\right\}$. Invoking Hölder's inequality, (i) of Lemma 2.2, the property of $\omega \in A_{\frac{q_{1}}{r^{\prime}}}$ and the reverse Hölder inequality, we have that

$$
\begin{aligned}
& \mathrm{u}_{1}^{\prime} \leqslant \mathrm{C} 2^{-\mathrm{kn}+\mathrm{k \mu}}\left(\int _ { \mathrm { E } _ { \mathrm { k } } } | \mathrm { b } ( \mathrm { x } ) - \mathrm { b } _ { \mathrm { B } _ { \mathrm { k } } } | ^ { \mathrm { q } _ { 2 } } \left(\left(\int_{\mathrm{E}_{j}}|\Omega(x-y)|^{\mathrm{q}_{1}^{\prime}} w(y)^{-\frac{\mathrm{q}_{1}^{\prime}}{\mathrm{q}_{1}}} d y\right)^{\frac{1}{q_{1}^{\prime}}}\right.\right. \\
& \left.\left.\times\left(\int_{E_{j}}|f(y)|^{q_{1}} \omega(y) d y\right)^{\frac{1}{q_{1}}}\right)^{q_{2}} \omega^{1-\left(1-\frac{\mu}{n}\right) q_{2}}(x) d x\right)^{\frac{1}{q_{2}}} \\
& \leqslant C 2^{-k n+k \mu}\left\|f \chi_{E_{j}}\right\|_{L^{q_{1}}\left(\mathbb{R}^{n}, w\right)}\left(\int _ { E _ { k } } | b ( x ) - b _ { B _ { k } } | ^ { q _ { 2 } } \left(\left(\int_{E_{j}}|\Omega(x-y)|^{r} d y\right)^{\frac{1}{r}}\right.\right. \\
& \left.\left.\times\left(\int_{B_{j}} \omega(y)^{-\frac{q_{1}^{\prime}}{q_{1}}\left(\frac{r}{q_{1}^{\prime}}\right)^{\prime}} d y\right)^{\frac{1}{q_{1}^{\prime}}-\frac{1}{r}}\right)^{q_{2}} \omega^{1-\left(1-\frac{\mu}{n}\right) q_{2}}(x) d x\right)^{\frac{1}{q_{2}}} \\
& \leqslant C 2^{-k n+k \mu}\left\|f \chi_{E_{j}}\right\|_{L^{q_{1}}\left(\mathbb{R}^{n}, \omega\right)}\|\Omega\|_{L^{r}\left(S^{n-1}\right)} 2^{\frac{k(n-1)}{r}+\frac{j}{r}}\left(\int_{B_{j}} \omega(y)^{-\frac{1}{q_{1}-\frac{q_{1}}{r}-1}} d y\right)^{\frac{1}{q_{1}^{\prime}-\frac{1}{r}}} \\
& \times\left(\int_{B_{k}}\left|b(x)-b_{B_{k}}\right|^{q_{2}} w^{1-\left(1-\frac{\mu}{n}\right) q_{2}}(x) d x\right)^{\frac{1}{q_{2}}} \\
& \leqslant C 2^{-k n+k \mu}\left\|f \chi_{E_{j}}\right\|_{L^{q_{1}}\left(\mathbb{R}^{n}, \omega\right)}\|\Omega\|_{L^{r}\left(S^{n-1}\right)} 2^{\frac{k(n-1)}{r}+\frac{j}{r}} \omega\left(B_{j}\right)^{-\frac{1}{q_{1}}}\left|B_{j}\right|^{1-\frac{1}{r}} \\
& \times\left(\int_{B_{k}}\left|b(x)-b_{B_{k}}\right|^{s} \omega^{1-s}(x) d x\right)^{\frac{1}{s}}\left(\int_{B_{k}} \omega^{\left(1-\left(1-\frac{\mu}{n}\right) q_{2}-\frac{\mathrm{q}_{2}(1-s)}{s}\right)\left(\frac{s}{q_{2}}\right)^{\prime}}(x) d x\right)^{\frac{1}{q_{2}}-\frac{1}{s}} \\
& \leqslant C 2^{(j-k)\left(n-\frac{n}{r}+\frac{1}{r}\right)} 2^{k \mu}\left\|f \chi_{E_{j}}\right\|_{L^{q_{1}}\left(\mathbb{R}^{n}, \omega\right)}\|\Omega\|_{L^{r}\left(S^{n-1}\right)} \omega\left(B_{j}\right)^{-\frac{1}{q_{1}}}\|b\|_{L_{\beta_{3, \omega}^{s}}^{s}} \omega\left(B_{k}\right)^{\frac{\beta}{n}+\frac{1}{s}} \\
& \times \omega\left(B_{k}\right)^{\frac{1}{q_{2}}-\frac{1}{s}+\frac{\mu}{n}}\left|B_{k}\right|^{-\frac{\mu}{n}}
\end{aligned}
$$

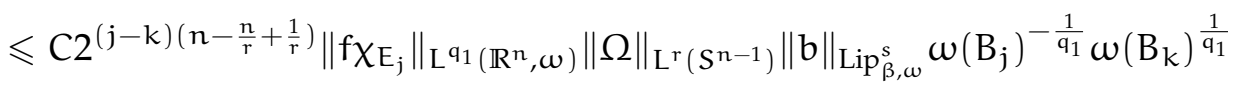




$$
\leqslant C 2^{(j-k) n\left(\frac{1}{q_{1}^{\prime}}-\frac{1}{r}+\frac{1}{n r}\right)}\|\Omega\|_{L^{r}\left(S^{n-1}\right)}\left\|f \chi_{E_{j}}\right\|_{L^{q_{1}}\left(\mathbb{R}^{n}, \omega\right)}\|b\|_{\operatorname{Lip}_{\beta, \omega}^{q_{2}}} .
$$

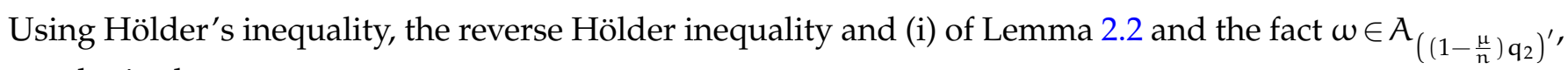
we obtain that

$$
\begin{aligned}
& u_{2}^{\prime}=C 2^{-k n+k \mu}\left(\int_{E_{k}}\left(\int_{E_{j}}\left|\Omega(x-y)\left\|b_{B_{k}}-b(y)\right\| f(y)\right| d y\right)^{q_{2}} w^{1-\left(1-\frac{\mu}{n}\right) q_{2}}(x) d x\right)^{\frac{1}{q_{2}}} \\
& \leqslant C 2^{-k n+k \mu}\left(\int_{E_{k}}\left(\int_{E_{j}}|\Omega(x-y)|^{q_{1}^{\prime}}\left|b_{B_{k}}-b(y)\right|^{q_{1}^{\prime}} w(y)^{-\frac{q_{1}^{\prime}}{q_{1}}} d y\right)^{\frac{q_{2}}{q_{1}^{\prime}}}\right. \\
& \left.\times\left(\int_{E_{j}}|f(y)|^{q_{1}} w(y) d y\right)^{\frac{q_{2}}{q_{1}}} \omega(x)^{1-\left(1-\frac{\mu}{n}\right) q_{2}} d x\right)^{\frac{1}{q_{2}}} \\
& \leqslant C 2^{-k n+k \mu}\left\|f \chi_{E_{j}}\right\|_{L^{q_{1}}\left(\mathbb{R}^{n}, w\right)}\left(\int_{E_{k}}\left(\int_{E_{j}}|\Omega(x-y)|^{r} d y\right)^{\frac{q_{2}}{r}}\left(\int_{B_{j}}\left|b(y)-b_{B_{k}}\right|^{s} \omega(y)^{1-s} d y\right)^{\frac{q_{2}}{s}}\right.
\end{aligned}
$$

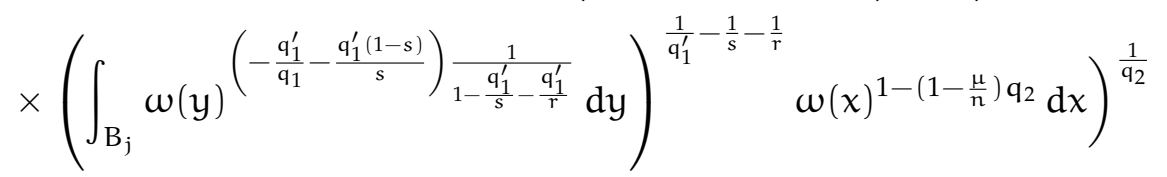

$$
\begin{aligned}
& \leqslant C 2^{-k n+k \mu} 2^{\frac{k(n-1)+j}{r}}\left\|f \chi_{E_{j}}\right\|_{L^{q_{1}}\left(\mathbb{R}^{n}, \omega\right)}\|\Omega\|_{L^{r}\left(S^{n-1}\right)}(k-j)\|b\|_{L i p_{\beta, \omega}^{s}} \omega\left(B_{j}\right)^{\frac{\beta}{n}+\frac{1}{s}}\left|B_{j}\right|^{-\frac{1}{r}} \omega\left(B_{j}\right)^{\frac{1}{q_{1}^{\prime}}-\frac{1}{s}} \\
& \times\left(\int_{B_{k}} \omega(x)^{1-\left(1-\frac{\mu}{n}\right) q_{2}} d x\right)^{\frac{1}{q_{2}}} \\
& \leqslant C(k-j) 2^{-k n+k \mu} 2^{(j-k)\left(\frac{1}{r}-\frac{n}{r}\right)}\left\|f \chi_{E_{j}}\right\|_{L^{q_{1}}\left(\mathbb{R}^{n}, w\right)}\|\Omega\|_{L^{r}\left(S^{n-1}\right)}\|b\|_{\operatorname{Lip}_{\beta, \omega}^{s}} \omega\left(B_{j}\right)^{\frac{\beta}{n}+\frac{1}{q_{1}^{\prime}}} \\
& \times\left|B_{k}\right|^{1-\frac{\mu}{n}} \omega\left(B_{k}\right)^{\frac{1}{q_{2}}-1+\frac{\mu}{n}} \\
& \leqslant C(k-j) 2^{(j-k) n\left(\frac{1}{n r}-\frac{1}{r}\right)}\|\Omega\|_{L^{r}\left(S^{n-1}\right)}\left\|f \chi_{E_{j}}\right\|_{L^{q_{1}}\left(\mathbb{R}^{n}, \omega\right)}\|b\|_{\operatorname{Lip}_{\beta, \omega}^{s}} \omega\left(B_{k}\right)^{\frac{1}{q_{2}}-1+\frac{\mu}{n}}
\end{aligned}
$$

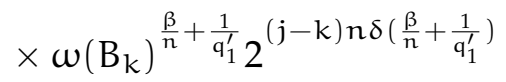

$$
\begin{aligned}
& \leqslant C(k-j) 2^{(j-k) n\left(\frac{\beta \delta}{n}+\frac{\delta}{q_{1}^{\prime}}+\frac{1}{n r}-\frac{1}{r}\right)}\|\Omega\|_{L^{r}\left(S^{n-1}\right)}\left\|f \chi_{E_{j}}\right\|_{L^{q_{1}}\left(\mathbb{R}^{n}, \omega\right)}\|b\|_{\operatorname{Lip}_{\beta, \omega^{\prime}}^{s}}
\end{aligned}
$$

which, together with the estimate of $\mathrm{U}_{1}^{\prime}$, concludes that

$$
\left\|\left[b, T_{\mu}\right] f_{j} \chi_{k}\right\|_{L^{q_{2}}\left(\mathbb{R}^{n}, \omega^{\left.1-\left(1-\frac{\mu}{n}\right) q_{2}\right)}\right.} \leqslant C(k-j) 2^{(j-k) n\left(\min \left\{\frac{1}{q_{1}^{\prime}}, \delta\left(\frac{\beta}{n}+\frac{1}{q_{1}^{\prime}}\right)\right\}+\frac{1}{n r}-\frac{1}{r}\right)}\left\|f_{E_{j}}\right\|_{L^{q_{1}}\left(\mathbb{R}^{n}, w\right)} .
$$

The combination of the above estimate with Lemma 2.6 and (ii) of Lemma 2.5 derives that

$$
\begin{aligned}
I_{1} \leqslant & C \omega(B)^{-p \lambda} \sum_{k=-\infty}^{0} \omega\left(E_{k}\right)^{\alpha p}\left(\sum_{j=-\infty}^{k-2}(k-j) 2^{(j-k) n\left(\min \left\{\frac{1}{q_{1}^{\prime}}, \delta\left(\frac{\beta}{n}+\frac{1}{q_{1}^{\prime}}\right)\right\}+\frac{1}{n r}-\frac{1}{r}\right)}\left\|f \chi_{E_{j}}\right\|_{L^{q_{1}}\left(\mathbb{R}^{n}, \omega\right)}\right)^{p} \\
\leqslant & C \omega(B)^{-p \lambda} \sum_{k=-\infty}^{0} \omega\left(E_{k}\right)^{\alpha p}\left(\sum_{j=-\infty}^{k-2}(k-j) 2^{(j-k) n\left(\min \left\{\frac{1}{q_{1}^{\prime}}, \delta\left(\frac{\beta}{n}+\frac{1}{q_{1}^{\prime}}\right)\right\}+\frac{1}{n r}-\frac{1}{r}\right)} \omega\left(E_{j}\right)^{-\alpha+\lambda}\right. \\
& \left.\times\left(\omega\left(E_{j}\right)^{-\lambda p+\alpha p}\left\|f \chi_{E_{j}}\right\|_{L^{q_{1}}\left(\mathbb{R}^{n}, \omega\right)}^{p}\right)^{\frac{1}{p}}\right)^{p} \\
\leqslant & C\|f\|_{L_{p_{,}, q_{1}}^{\alpha,}\left(\mathbb{R}^{n}, \omega, \omega\right)}^{p} \omega(B)^{-p \lambda} \sum_{k=-\infty}^{0} \omega\left(E_{k}\right)^{\alpha p}
\end{aligned}
$$




$$
\begin{aligned}
& \quad\left\{\begin{array}{l}
\left(\sum_{j=-\infty}^{k-2}(k-j) 2^{(j-k) n\left(\min \left\{\frac{1}{q_{1}^{\prime}}, \delta\left(\frac{\beta}{n}+\frac{1}{q_{1}^{\prime}}\right)\right\}+\frac{1}{n r}-\frac{1}{r}\right)} 2^{n(j-k)(\lambda-\alpha)} \omega\left(E_{k}\right)^{\lambda-\alpha}\right)^{p}, \quad \alpha \geqslant \lambda \\
\left(\sum_{j=-\infty}^{k-2}(k-j) 2^{(j-k) n\left(\min \left\{\frac{1}{q_{1}^{\prime}}, \delta\left(\frac{\beta}{n}+\frac{1}{q_{1}^{\prime}}\right)\right\}+\frac{1}{n r}-\frac{1}{r}\right)} 2^{n(j-k) \delta(\lambda-\alpha)} \omega\left(E_{k}\right)^{\lambda-\alpha}\right)^{p}, \quad \alpha<\lambda
\end{array}\right. \\
& \leqslant C\|f\|_{L_{p, q_{1}}^{\alpha, \lambda}\left(\mathbb{R}^{n}, \omega, \omega\right)}^{p} \omega(B)^{-p \lambda} \sum_{k=-\infty}^{0} \omega\left(E_{k}\right)^{\lambda p} \\
& \quad \times\left\{\begin{array}{l}
\left(\sum_{j=-\infty}^{k-2}(k-j) 2^{(j-k) n\left(\min \left\{\frac{1}{q_{1}^{\prime}}, \delta\left(\frac{\beta}{n}+\frac{1}{q_{1}^{\prime}}\right)\right\}+\frac{1}{n r}-\frac{1}{r}+\lambda-\alpha\right)}\right)^{p}, \quad \alpha \geqslant \lambda \\
\left(\sum_{j=-\infty}^{k-2}(k-j) 2^{(j-k) n\left(\min \left\{\frac{1}{q_{1}^{\prime}}, \delta\left(\frac{\beta}{n}+\frac{1}{q_{1}^{\prime}}\right)\right\}+\frac{1}{n r}-\frac{1}{r}+\lambda \delta-\alpha \delta\right)}\right)^{p}, \quad \alpha<\lambda
\end{array}\right. \\
& \leqslant C\|f\|_{L_{,, q_{1}}^{\alpha, \lambda}\left(\mathbb{R}^{n}, \omega, \omega\right)^{\prime}}^{p}
\end{aligned}
$$

where we require either $\lambda \leqslant \alpha<\lambda+\frac{1}{n r}-\frac{1}{r}+\min \left\{\frac{1}{q_{1}^{\prime}}, \delta\left(\frac{\beta}{n}+\frac{1}{q_{1}^{\prime}}\right)\right\}$ or $\alpha<\lambda$.

Finally, we estimate $I_{3}$. When $x \in E_{k}, y \in E_{j}$ and $j \geqslant k+2$, then $|x-y| \sim\left|x-x_{0}\right| \sim 2^{j} d$. If $r>$ $\max \left\{q_{2}, \frac{q_{1}(n-1)}{n \delta-q_{1} \mu}\right\}$, Minkowski's inequality gives that

$$
\begin{aligned}
& \left\|\left[b, T_{\mu}\right] f_{j} \chi_{k}\right\|_{L^{q_{2}}\left(\mathbb{R}^{n}, \omega^{1-\left(1-\frac{\mu}{n}\right) q_{2}}\right)} \\
& \leqslant C 2^{-j n+j \mu}\left(\int_{E_{k}}\left(\int_{E_{j}}|\Omega(x-y)\|b(x)-b(y)\| f(y)| d y\right)^{\mathbf{q}_{2}} \omega^{1-\left(1-\frac{\mu}{n}\right) q_{2}}(x) d x\right)^{\frac{1}{q_{2}}} \\
& \leqslant C 2^{-j n+j \mu} \int_{E_{j}}\left(\int_{E_{k}}|\Omega(x-y)|^{q_{2}}\left|b(x)-b_{B_{k}}\right|^{q_{2}} w^{1-\left(1-\frac{\mu}{n}\right) q_{2}}(x) d x\right)^{\frac{1}{q_{2}}}|f(y)| d y \\
& +C 2^{-j n+j \mu} \int_{E_{j}}\left(\int_{E_{k}}|\Omega(x-y)|^{q_{2}} w^{1-\left(1-\frac{\mu}{n}\right) q_{2}}(x) d x\right)^{\frac{1}{q_{2}}}\left|b_{B_{k}}-b(y)\right| f(y) \mid d y \\
& :=\mathrm{C}\left(\mathrm{V}_{1}+\mathrm{V}_{2}\right) \text {. }
\end{aligned}
$$

Choosing $s>\max \left\{\frac{\mathrm{q}_{2} r}{r-q_{2}}, q_{1}^{\prime}\right\}$ and by the same argument to bound $\mathrm{U}_{1}$ and $\mathrm{U}_{2}$, we get that

$$
\begin{aligned}
& V_{1} \leqslant C 2^{-j n+j \mu} \int_{E_{j}}\left(\int_{E_{k}}|\Omega(x-y)|^{r} d x\right)^{\frac{1}{r}}\left(\int_{B_{k}}\left|b(x)-b_{B_{k}}\right|^{s} \omega^{1-s}(x) d x\right)^{\frac{1}{s}}
\end{aligned}
$$

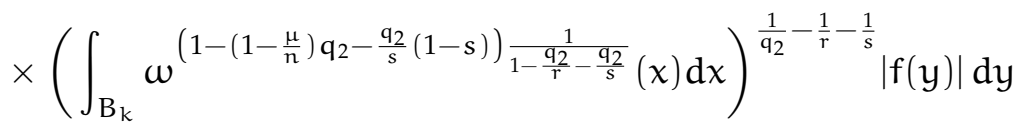

$$
\begin{aligned}
& \leqslant C 2^{-j n+j \mu}\|\Omega\|_{L^{r}\left(S^{n-1}\right)} 2^{\frac{j(n-1)}{r}+\frac{k}{r}}\|b\|_{\operatorname{Lip}_{\beta, \omega}^{s}} \omega\left(B_{k}\right)^{\frac{\beta}{n}+\frac{1}{s}} \omega\left(B_{k}\right)^{\frac{1}{q_{2}}-\frac{1}{s}+\frac{\mu}{n}}\left|B_{k}\right|^{-\frac{1}{r}-\frac{\mu}{n}} \\
& \times\left\|f \chi_{E_{j}}\right\|_{L^{q_{1}\left(\mathbb{R}^{n}, \omega\right)}}\left|B_{j}\right| \omega\left(B_{j}\right)^{-\frac{1}{q_{1}}} \\
& \leqslant C 2^{(j-k) n\left(\frac{1}{r}+\frac{\mu}{n}-\frac{1}{n r}\right)} \Omega\left\|_{L^{r}\left(S^{n-1}\right)}\right\| b\left\|_{\operatorname{Lip}_{\beta, \omega}^{s}}\right\| f \chi_{E_{j}} \|_{L^{q_{1}}\left(\mathbb{R}^{n}, \omega\right)} \omega\left(B_{j}\right)^{\frac{1}{q_{1}}}\left(\frac{\left|B_{k}\right|}{\left|B_{j}\right|}\right)^{\frac{\delta}{q_{1}}} \omega\left(B_{j}\right)^{-\frac{1}{q_{1}}} \\
& \leqslant C 2^{(k-j) n\left(\frac{\delta}{q_{1}}-\frac{\mu}{n}-\frac{1}{r}+\frac{1}{n r}\right)} \Omega\left\|_{L^{r}\left(S^{n-1}\right)}\right\| b\left\|_{\operatorname{Lip}_{\beta, \omega}^{s}}\right\| f \chi_{E_{j}} \|_{L^{q_{1}}\left(\mathbb{R}^{n}, \omega\right)},
\end{aligned}
$$

and

$$
V_{2} \leqslant C 2^{-j n+j \mu} \int_{E_{j}}\left(\int_{E_{k}}|\Omega(x-y)|^{r} d x\right)^{\frac{1}{r}}\left(\int_{B_{k}} \omega^{\left(1-\left(1-\frac{\mu}{n}\right) q_{2}\right)\left(\frac{r}{q_{2}}\right)^{\prime}}(x) d x\right)^{\frac{1}{q_{2}}-\frac{1}{r}}\left|b_{B_{k}}-b(y) \| f(y)\right| d y
$$




$$
\begin{aligned}
\leqslant & C 2^{-j n+j \mu}\|\Omega\|_{L^{r}\left(S^{n-1}\right)} 2^{\frac{j(n-1)}{r}+\frac{k}{r}} \omega\left(B_{k}\right)^{\frac{1}{q_{2}}-1+\frac{\mu}{n}}\left|B_{k}\right|^{1-\frac{\mu}{n}-\frac{1}{r}}\left\|f \chi_{E_{j}}\right\|_{L^{q_{1}}\left(\mathbb{R}^{n}, \omega\right)} \\
& \times(j-k) \omega\left(B_{j}\right)^{\frac{\beta}{n}+\frac{1}{s}}\|b\|_{L_{p}^{s} s, \omega} \omega\left(B_{j}\right)^{1-\frac{1}{q_{1}}-\frac{1}{s}} \\
\leqslant & C(j-k) 2^{(j-k) n\left(\frac{1}{r}+\frac{\mu}{n}-\frac{1}{n r}-1\right)}\|\Omega\|_{L^{r}\left(S^{n-1}\right)}\left\|f \chi_{E_{j}}\right\|_{L^{q_{1}}\left(\mathbb{R}^{n}, \omega\right)}\|b\|_{L_{i j}^{s} p_{\beta, \omega}^{s}} \omega\left(B_{k}\right)^{\frac{1}{q_{2}}-1+\frac{\mu}{n}} \omega\left(B_{j}\right)^{1+\frac{\beta}{n}-\frac{1}{q_{1}}} \\
\leqslant & C(j-k) 2^{(j-k) n\left(\frac{1}{r}+\frac{\mu}{n}-\frac{1}{n r}-1\right)}\|\Omega\|_{L^{r}\left(S^{n-1}\right)}\left\|f \chi_{E_{j}}\right\|_{L^{q_{1}}\left(\mathbb{R}^{n}, \omega\right)}\|b\|_{L_{\beta, \omega}^{s}} \omega\left(B_{k}\right)^{\frac{1}{q_{2}}-1+\frac{\mu}{n}} \\
& \times \omega\left(B_{k}\right)^{1+\frac{\beta}{n}-\frac{1}{q_{1}}} 2^{(j-k) n\left(\frac{\beta}{n}+1-\frac{1}{q_{1}}\right)} \\
\leqslant & C(j-k) 2^{(k-j) n\left(\frac{1}{q_{2}}-\frac{1}{r}+\frac{1}{n r}\right)}\|\Omega\|_{L^{r}\left(S^{n-1}\right)}\left\|f \chi_{E_{j}}\right\|_{L^{q_{1}}\left(\mathbb{R}^{n}, \omega\right)}\|b\|_{\operatorname{Lip}_{\beta, \omega}^{s}} .
\end{aligned}
$$

Thus,

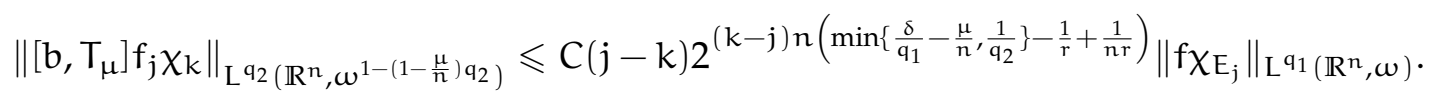

Therefore, we can get by Lemma 2.6 and (i) of Lemma 2.5 that

$$
\begin{aligned}
& I_{3} \leqslant C \omega(B)^{-p} \lambda \sum_{k=-\infty}^{0} \omega\left(E_{k}\right)^{\alpha p}\left(\sum_{j=k+2}^{\infty}(j-k) 2^{(k-j) n\left(\min \left\{\frac{\delta}{q_{1}}-\frac{\mu}{n}, \frac{1}{q_{2}}\right\}-\frac{1}{r}+\frac{1}{n r}\right)}\left\|f \chi_{E_{j}}\right\|_{L^{q_{1}}\left(\mathbb{R}^{n}, \omega\right)}\right)^{p} \\
& \leqslant \mathrm{C} \omega(B)^{-p \lambda} \sum_{k=-\infty}^{0} \omega\left(E_{k}\right)^{\alpha p}\left(\sum_{j=k+2}^{\infty}(j-k) 2^{(k-j) n\left(\min \left\{\frac{\delta}{q_{1}}-\frac{\mu}{n}, \frac{1}{q_{2}}\right\}-\frac{1}{r}+\frac{1}{n r}\right)} \omega\left(E_{j}\right)^{-\alpha+\lambda}\right. \\
& \left.\times\left(\omega\left(E_{j}\right)^{-\lambda p+\alpha p}\left\|f \chi_{E_{j}}\right\|_{L^{q_{1}}\left(\mathbb{R}^{n}, \omega\right)}^{p}\right)^{\frac{1}{p}}\right)^{p} \\
& \leqslant C\|f\|_{L_{p, q_{1}}^{\alpha, \lambda}\left(\mathbb{R}^{n}, \omega, \omega\right)}^{p} \omega(B)^{-p \lambda} \sum_{k=-\infty}^{0} \omega\left(E_{k}\right)^{\alpha p} \\
& \times\left\{\begin{array}{l}
\left(\sum_{j=k+2}^{\infty}(j-k) 2^{(k-j) n\left(\min \left\{\frac{\delta}{q_{1}}-\frac{\mu}{n}, \frac{1}{q_{2}}\right\}-\frac{1}{r}+\frac{1}{n r}\right)} 2^{n(j-k) \delta(\lambda-\alpha)} \omega\left(E_{k}\right)^{\lambda-\alpha}\right)^{p}, \quad \alpha \geqslant \lambda \\
\left(\sum_{j=k+2}^{\infty}(j-k) 2^{(k-j) n\left(\min \left\{\frac{\delta}{q_{1}}-\frac{\mu}{n}, \frac{1}{q_{2}}\right\}-\frac{1}{r}+\frac{1}{n r}\right)} 2^{n(j-k)(\lambda-\alpha)} \omega\left(E_{k}\right)^{\lambda-\alpha}\right)^{p}, \quad \alpha<\lambda
\end{array}\right. \\
& \leqslant C\|f\|_{L_{p, q_{1}}^{\alpha, \lambda}\left(\mathbb{R}^{n}, \omega, \omega\right)}^{p} \omega(B)^{-p \lambda} \sum_{k=-\infty}^{0} \omega\left(E_{k}\right)^{\lambda p} \\
& \times\left\{\begin{array}{l}
\left(\sum_{j=k+2}^{\infty}(j-k) 2^{(k-j) n\left(\min \left\{\frac{\delta}{q_{1}}-\frac{\mu}{n}, \frac{1}{q_{2}}\right\}-\frac{1}{r}+\frac{1}{n r}-\lambda \delta+\alpha \delta\right)}\right)^{p}, \quad \alpha \geqslant \lambda \\
\left(\sum_{j=k+2}^{\infty}(j-k) 2^{(k-j) n\left(\min \left\{\frac{\delta}{q_{1}}-\frac{\mu}{n}, \frac{1}{q_{2}}\right\}-\frac{1}{r}+\frac{1}{n r}-\lambda+\alpha\right)}\right)^{p}, \quad \alpha<\lambda
\end{array}\right. \\
& \leqslant C\|f\|_{L_{p, q_{1}}^{\alpha, \lambda}\left(\mathbb{R}^{n}, \omega, \omega\right)^{\prime}}^{p}
\end{aligned}
$$

where we need either $\alpha \geqslant \lambda$ or $\lambda+\frac{1}{r}-\frac{1}{n r}-\min \left\{\frac{\delta}{q_{1}}-\frac{\mu}{n}, \frac{1}{q_{2}}\right\}<\alpha<\lambda$.

If $r>\max \left\{q_{1}^{\prime}, \frac{q_{1}^{\prime}(n-1)}{\left(q_{1}^{\prime} \beta+n\right) \delta}\right\}$, we decompose

$$
\begin{aligned}
& \left\|\left[b, T_{\mu}\right] f_{j} \chi_{k}\right\|_{L_{2}\left(\mathbb{R}^{n}, \omega^{1-\left(1-\frac{\mu}{n}\right) q_{2}}\right)} \\
& \leqslant C 2^{-j n+j \mu}\left(\int_{E_{k}}\left(\int_{E_{j}}|\Omega(x-y)\|b(x)-b(y)\| f(y)| d y\right)^{q_{2}} \omega^{1-\left(1-\frac{\mu}{n}\right) q_{2}}(x) d x\right)^{\frac{1}{q_{2}}}
\end{aligned}
$$




$$
\begin{aligned}
\leqslant & C 2^{-j n+j \mu}\left(\int_{E_{k}}\left|b(x)-b_{B_{k}}\right| q_{2}\left(\int_{E_{j}}|\Omega(x-y) \| f(y)| d y\right)^{q_{2}} w^{1-\left(1-\frac{\mu}{n}\right) q_{2}}(x) d x\right)^{\frac{1}{q_{2}}} \\
& +C 2^{-j n+j \mu}\left(\int_{E_{k}}\left(\int_{E_{j}}\left|\Omega(x-y)\left\|b_{B_{k}}-b(y)\right\| f(y)\right| d y\right)^{q_{2}} \omega^{1-\left(1-\frac{\mu}{n}\right) q_{2}}(x) d x\right)^{\frac{1}{q_{2}}} \\
:= & C\left(V_{1}^{\prime}+V_{2}^{\prime}\right) .
\end{aligned}
$$

By (iii) of Lemma 2.2 and applying the same method as used in $\mathrm{U}_{1}^{\prime}$ and $\mathrm{U}_{2}^{\prime}$, we may prove that

$$
\begin{aligned}
& V_{1}^{\prime} \leqslant C 2^{-j n+j \mu}\left(\int _ { E _ { k } } | b ( x ) - b _ { B _ { k } } | ^ { q _ { 2 } } \left(\left(\int_{E_{j}}|\Omega(x-y)|^{q_{1}^{\prime}} \omega(y)^{-\frac{q_{1}^{\prime}}{q_{1}}} d y\right)^{\frac{1}{q_{1}^{\prime}}}\right.\right. \\
& \left.\left.\times\left(\int_{E_{j}}|f(y)|^{q_{1}} \omega(y) d y\right)^{\frac{1}{q_{1}}}\right)^{q_{2}} \omega^{1-\left(1-\frac{\mu}{n}\right) q_{2}}(x) d x\right)^{\frac{1}{q_{2}}} \\
& \leqslant C 2^{-j n+j \mu}\left\|f \chi_{E_{j}}\right\|_{L^{q_{1}}\left(\mathbb{R}^{n}, \omega\right)}\|\Omega\|_{L^{r}\left(S^{n-1}\right)} 2^{\frac{j n}{r}} \omega\left(B_{j}\right)^{-\frac{1}{q_{1}}}\left|B_{j}\right|^{1-\frac{1}{r}}\|b\|_{L i p_{\beta, \omega}^{s}} \\
& \times \omega\left(B_{k}\right)^{\frac{\beta}{n}+\frac{1}{s}} \omega\left(B_{k}\right)^{\frac{1}{q_{2}}-\frac{1}{s}+\frac{\mu}{n}}\left|B_{k}\right|^{-\frac{\mu}{n}}
\end{aligned}
$$

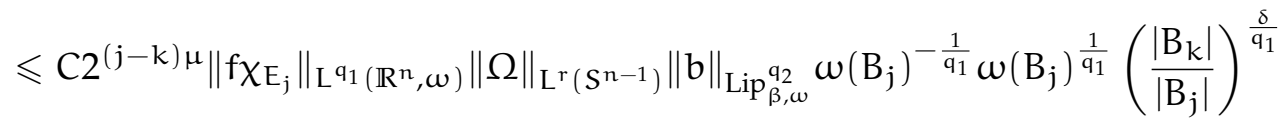

$$
\begin{aligned}
& \leqslant C 2^{(k-j) n\left(\frac{\delta}{q_{1}}-\frac{\mu}{n}\right)}\|\Omega\|_{L^{r}\left(S^{n-1}\right)}\left\|f \chi_{E_{j}}\right\|_{L^{q_{1}}\left(\mathbb{R}^{n}, \omega\right)}\|b\|_{L_{p_{\beta, \omega}}^{q_{2}},}
\end{aligned}
$$

and

$$
\begin{aligned}
& V_{2}^{\prime}=C 2^{-j n+j \mu}\left(\int_{E_{k}}\left(\int_{E_{j}}\left|\Omega(x-y)\left\|b_{B_{k}}-b(y)\right\| f(y)\right| d y\right)^{q_{2}} w^{1-\left(1-\frac{\mu}{n}\right) q_{2}}(x) d x\right)^{\frac{1}{q_{2}}} \\
& \leqslant C 2^{-j n+j \mu}\left(\int_{E_{k}}\left(\int_{E_{j}}|\Omega(x-y)|^{q_{1}^{\prime}}\left|b_{B_{k}}-b(y)\right|^{q_{1}^{\prime}} \omega(y)^{-\frac{q_{1}^{\prime}}{q_{1}}} d y\right)^{\frac{q_{2}}{q_{1}^{\prime}}}\left(\int_{E_{j}}|f(y)|^{q_{1}} \omega(y) d y\right)^{\frac{q_{2}}{q_{1}}}\right. \\
& \left.\times \omega(x)^{1-\left(1-\frac{\mu}{n}\right) q_{2}} d x\right)^{\frac{1}{q_{2}}} \\
& \leqslant C 2^{-j n+j \mu}\left\|f \chi_{E_{j}}\right\|_{L^{q_{1}}\left(\mathbb{R}^{n}, \omega\right)} 2^{\frac{j n}{r}}\|\Omega\|_{L^{r}\left(S^{n-1}\right)}(j-k)\|b\|_{L i p_{\beta, \omega}^{s}} \omega\left(B_{j}\right)^{\frac{\beta}{n}+\frac{1}{s}}\left|B_{j}\right|^{-\frac{1}{r}} \omega\left(B_{j}\right)^{\frac{1}{q_{1}^{\prime}}-\frac{1}{s}} \\
& \times\left|B_{k}\right|^{1-\frac{\mu}{n}} \omega\left(B_{k}\right)^{\frac{1}{q_{2}}-1+\frac{\mu}{n}}
\end{aligned}
$$

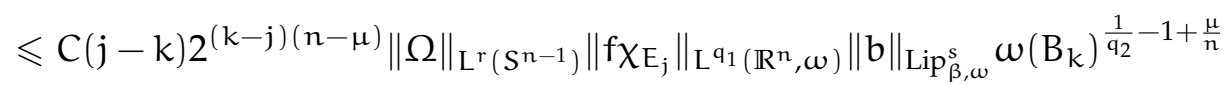

$$
\begin{aligned}
& \times \omega\left(B_{k}\right)^{1-\frac{1}{q_{1}}+\frac{\beta}{n}} 2^{(j-k) n\left(1-\frac{1}{q_{1}}+\frac{\beta}{n}\right)} \\
& \leqslant C(j-k) 2^{(k-j) n \frac{1}{q_{2}}}\|\Omega\|_{L^{r}\left(S^{n-1}\right)}\left\|f \chi_{E_{j}}\right\|_{L^{q_{1}\left(\mathbb{R}^{n}, \omega\right)}}\|b\|_{\operatorname{Lip}_{\beta, \omega}^{s}} \text {. }
\end{aligned}
$$

Thus,

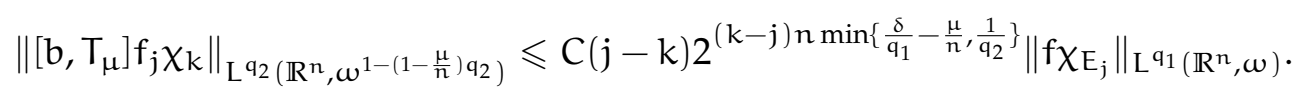

Applying Lemma 2.6 and (i) of Lemma 2.5 now yields

$$
\begin{aligned}
I_{3} & \leqslant C \omega(B)^{-p \lambda} \sum_{k=-\infty}^{0} \omega\left(E_{k}\right)^{\alpha p}\left(\sum_{j=k+2}^{\infty}(j-k) 2^{(k-j) n \min \left\{\frac{\delta}{q_{1}}-\frac{\mu}{n}, \frac{1}{q_{2}}\right\}}\left\|f \chi_{E_{j}}\right\|_{L^{q_{1}}\left(\mathbb{R}^{n}, \omega\right)}\right)^{p} \\
& \leqslant C \omega(B)^{-p \lambda} \sum_{k=-\infty}^{0} \omega\left(E_{k}\right)^{\alpha p}\left(\sum_{j=k+2}^{\infty}(j-k) 2^{(k-j) n \min \left\{\frac{\delta}{q_{1}}-\frac{\mu}{n}, \frac{1}{q_{2}}\right\}} \omega\left(E_{j}\right)^{-\alpha+\lambda}\right.
\end{aligned}
$$




$$
\begin{aligned}
& \left.\quad \times\left(\omega\left(E_{j}\right)^{-\lambda p+\alpha p}\left\|f \chi_{E_{j}}\right\|_{L^{q_{1}}\left(\mathbb{R}^{n}, \omega\right)}^{p}\right)^{\frac{1}{p}}\right)^{p} \\
& \leqslant \\
& \leqslant
\end{aligned}
$$

where we demand either $\alpha \geqslant \lambda$ or $\lambda-\min \left\{\frac{\delta}{q_{1}}-\frac{\mu}{n}, \frac{1}{q_{2}}\right\}<\alpha<\lambda$.

\section{Conclusion}

In this paper, we have obtained bounded criterion for commutators of some sublinear operators on weighted Morrey spaces. Utilizing these criteria, we have obtained the boundedness of the commutators associated with some classic operators such as singular integral, fractional integral, Marcinkiwicz integral etc. on weighted Morrey spaces. The results derived in this paper become natural generalizations of classical results. It is expected that the interested reader may find useful applications of these results and consequently this paper may stimulate further research in this area.

\section{Acknowledgment}

The author is thankful to the anonymous referee for his/her valuable comments and suggestions. The author is supported by the NNSF of China $(11461033,11400129)$ and the foundation of CSC $(201608360133)$.

\section{References}

[1] J. Carcía-Cuerva, J. L. Rubio de Francia, Weighted norm inequalities and related topics, North-Holland, Amsterdam, (1985). 2

[2] Y. Ding, D. Yang, Z. Zhou, Boundedness of sublinear operators and commutators on $\mathrm{L}^{\mathrm{p}, \boldsymbol{\omega}}\left(\mathbb{R}^{\mathrm{n}}\right)$, Yokohama Math. J., 46 (1998), 15-27. 1

[3] D. Fan, S. Lu, D. Yang, Regularity in Morrey spaces of strong solutions to non-divergence elliptic equations with VMO coefficients, Georgian Math. J., 5 (1998), 425-440. 1

[4] G. Fazio, M. A. Ragusa, Interior estimates in Morrey spaces for strong solutions to nondivergence form equations with discontinuous coefficients, J. Funct. Anal., 112 (1993), 241-256. 1

[5] J. García-Cuerva, Weighted $\mathrm{H}^{p}$ spaces, Dissert. Math., 1979 (1979), 63 pages. 1, 1

[6] L. Grafakos, Classical and Modern Fourier Analysis, Pearson Education Inc., New Jersey, (2004). 2

[7] B. Hu, J. Gu, Necessary and sufficient conditions for boundedness of some commutators with weighted Lipschitz functions, J. Math. Anal. Appl., 340 (2008), 587-605. 1, 1

[8] Y. Komori, T. Mizuhara, Notes on Commutators and Morrey Spaces, Hokkaido Math. J., 32 (2003), 345-353. 1 
[9] Y. Komori, S. Shirai, Weighted Morrey spaces and a singular integral operator, Math. Nachr., 282 (2009), $219-231.1$

[10] Y. Lin, Strongly singular Calderón-Zygmund operator and commutator on Morrey type spaces, Acta Math. Sin., 23 (2007), 2097-2110. 1

[11] Y. Lin, G. Sun, Strongly singular Calderón-Zygmund operator and commutator on weighted Morrey type spaces, J. Ineq. Appl., 2014 (2014), 15 pages. 1.12

[12] S. Lu, Q. Wu, CBMO estimates for commutators and multilinear singular integrals, Math. Nachr., 276 (2004), 75-88. 2

[13] S. Lu, D. Yang, Z. Zhou, Sublinear operators with rough kernel on generalized Morrey spaces, Hokkaido Math. J., 27 (1998), 219-232. 1

[14] T. Mizuhara, Boundedness of some classical operators on generalized Morrey spaces, Springer, Tokyo, (1991). 1

[15] C. B. Morrey, On the solutions of quasi-linear elliptic partial differential equations, Trans. Amer. Math. Soc., 43 (1938), 126-166. 1

[16] S. Nakamura, Y. Sawano, The singular integral operator and its commutator on weighted Morrey spaces, Collect. Math., 68 (2017), 145-174. 1

[17] N. Samko, Weighted Hardy and singular operators in Morrey spaces, J. Math. Anal. Appl., 350 (2009), 56-72. 1

[18] Y. Sawano, H. Tanaka, Sharp maximal inequalities and commutators on Morrey spaces with non-doubling measures, Taiwanese J. Math., 11 (2007), 1091-1112. 1

[19] F. Soria, G. Weiss, A remark on singular integrals and power weights, Indiana Univ. Math. J., 43 (1994), 187-204. 1 